\title{
Observation of low-lying electronic states of NiD with multi-isotope analysis
}

\author{
Mahdi Abbasi ${ }^{1}$, Alireza Shayesteh ${ }^{1}$, Patrick Crozet $^{2}$, Amanda J. Ross ${ }^{2}$ \\ ${ }^{1}$ School of Chemistry, University of Tehran, I. R. Iran; ashayesteh@ut.ac.ir \\ ${ }^{2}$ Institut Lumière Matière (ILM), Université Lyon1 \& CNRS, France; amanda.ross@univ-lyon1.fr
}

Abstract.

Resolved laser induced fluorescence spectra of ${ }^{58 \mathrm{NiD}}$, recorded at Doppler resolution between 11000 and $18000 \mathrm{~cm}^{-1}$ have established more than 200 term energies in two of the three strongly interacting, low-lying states $\left({ }^{2} \Delta,{ }^{2} \Pi,{ }^{2} \Sigma^{+}\right)$of $\mathrm{NiD}$, associated with an $\mathrm{Ni}^{+}\left(3 \mathrm{~d}^{9}\right) \mathrm{D}^{-}$configuration. Our observations $\operatorname{span} v=0-5$ in the lowest spin-orbit component of the ground state, $\mathrm{X}^{2} \Delta_{5 / 2}, v=0-3$ in $\mathrm{X}^{2} \Delta_{3 / 2}$ and $v=0$ 1 in the lower component of the $\mathrm{W}^{2} \prod_{3 / 2}$ state. Collisional processes populate several neighbouring excited states, allowing us also to locate the first rotational levels of $\mathrm{A}(\Omega=5 / 2) v=1, \mathrm{I}(\Omega=3 / 2) v=0$ and $\mathrm{E}(\Omega=3 / 2) v=1$ at $16664.8,17367.3$ and $17508.1 \mathrm{~cm}^{-1}$ respectively. Spin-orbit and rotation-electronic interactions are strong in $\mathrm{NiD}$ (as in $\mathrm{NiH}$ ), and with no direct observation of either of the $\Omega^{\prime \prime}=1 / 2$ states, meaningful representations of the low-lying energy levels is difficult. We have therefore attempted a global, multi-isotope fit to reproduce the term energies of the known term energies of $\mathrm{NiD}$ and ${ }^{58,60,62} \mathrm{NiH}$ (where some $\Omega^{\prime \prime}=1 / 2$ levels are known). Dunham-type parameters have been used to represent the unperturbed ${ }^{2} \Delta$, ${ }^{2} \Pi$ and $2 \Sigma^{+}$states, with off-diagonal matrix elements (treating spin-orbit, $L$ - and $S$-uncoupling effects) based on $\mathrm{Ni}^{+}$atomic properties. Born-Oppenheimer breakdown terms were included in the model. The equilibrium bond lengths for ${ }^{58} \mathrm{NiH} /{ }^{58} \mathrm{NiD}$ are 1.4545(1)/1.4559(1) $\AA$ for the $\mathrm{X}^{2} \Delta$ state and 1.5094(2)/1.5083(2) $\AA$ for the $\mathrm{W}^{2} \Pi$ state.

Keywords

Laser-induced fluorescence; NiH; NiD; multi-isotope fit; spin-orbit perturbations, Supermultiplet Hamiltonian.

\section{INTRODUCTION}


One of the main motivations for high-resolution spectroscopic investigations of diatomic radicals such as $\mathrm{NiD}$ or $\mathrm{NiH}$ is to provide benchmark measurements for species whose electronic structure is challenging, but computationally tractable. In their review of transition metal species [1] Langhoff and Bauschlicher underlined the incentive to "develop methods capable not only of accurately describing small systems, but also for modeling larger systems". They illustrated this notion with the Ni atom, because of the problems posed by its near-degenerate low-lying states. Spectroscopy and quantum chemistry have advanced together, striving to understand and reproduce the effects of electron correlation in the bonds formed by transition metals, particularly in a context of materials of catalytic interest. Diatomic molecules such as $\mathrm{NiH}$ are the smallest building blocks of such systems. High level ab initio methods have become increasingly successful in reproducing molecular energy levels determined from gas-phase spectroscopy as basis set sizes (and computational resources) have grown [2-11]; the calculations on $\mathrm{NiH}$ by Marian [11,12], and by Zou and Liu [13], have proven particularly valuable in guiding assignments in our fluorescence spectra. Experimental determinations of the permanent electric dipole moment the $\mathrm{B}^{2} \Delta_{5 / 2}$ and $X$ ${ }^{2} \Delta_{5 / 2}$ states in $\mathrm{NiH}$ [14], have also provided stringent tests for theory [15-17]. NiH was chosen (Mayhall, ref. [5]) to highlight issues arising in coupled cluster calculations; it has also been used as a benchmark to test the performance of different density functionals, $[18,19]$ with a view to implementing less costly calculations allowing progression from diatomics to larger species. Reliable reference data are therefore important, and gas-phase spectroscopy, in its various forms, provides essential elements for quality control. In this context, we have studied some vibrationally excited levels of the three lowest-lying electronic states $\left({ }^{2} \Sigma^{+},{ }^{2} \Pi\right.$ and $\left.{ }^{2} \Delta\right)$ of NiD.

Electronic transitions in $\mathrm{NiH}$ and $\mathrm{NiD}$, formed in flames, vacuum furnace or in hollow cathode discharges, were first studied by photographic techniques [20-23]. The early work was 
reviewed by Scullman and co-workers in 1982, who photographed emission spectra of $\mathrm{NiH}$ [24] and $\mathrm{NiD}[25]$ to resolve some earlier discrepancies, and mapped out the pattern of vibrational levels in some ${ }^{2} \Delta$ and ${ }^{2} \Phi$ electronic states. Laser spectroscopy became increasingly important in the1980's. The Field group at MIT - often in collaboration with the Stockholm group - pursued investigations of the electronic states of $\mathrm{NiH}$ and $\mathrm{NiD}$ with a variety of laser excitation and fluorescence techniques [26,27], observing the $\mathrm{W}^{2} \Pi$ and $\mathrm{V}^{2} \Sigma^{+}$low-lying electronic states of $\mathrm{NiH}$ in dispersed fluorescence and depletion spectroscopy, and charting excited-state levels on $\mathrm{NiH}$ and NiD with $1.5 \leq \Omega^{\prime} \leq 3.5$, in the energy region $15500-19000 \mathrm{~cm}^{-1}$. In parallel, Brown and Urban and their collaborators studied the microwave and infrared transitions within the $\mathrm{X}^{2} \Delta$ ground electronic state of $\mathrm{NiH} / \mathrm{D}$ using laser magnetic resonance (LMR) techniques [28-32]; these measurements remain the best available to describe the parity splittings in the lowest electronic states of $\mathrm{NiD}$ and $\mathrm{NiH}$. Line-lists and combination differences from the electronic spectra reported in the 1980 's and early 1990 s enabled us to assign many of the bands in our resolved fluorescence spectra of NiD.

The unusual energy level patterns in $\mathrm{NiH}$ (and $\mathrm{NiD}$ ) are the consequence of strong spin-orbit interactions between close-lying electronic states. Zeeman spectra, extensively studied by the Field group [27,33-35], and later in Lyon [36], offer considerable insight into this mixing for the lower electronic states associated with ground state $\mathrm{Ni}^{+}$ion, confirming that $\Lambda$ and $\Sigma$ become ill-defined quantum numbers as molecular rotation increases. The so-called supermultiplet model, proposed by Gray et al. [26,37] and adopted by Marian in her theoretical work [11], was remarkably successful in explaining both the energy level structures and the rotational dependence of $g_{J}$ Landé factors in $v$ $\leq 2$ vibrational levels of the lowest ${ }^{2} \Sigma^{+},{ }^{2} \Pi$ and ${ }^{2} \Delta$ states of $\mathrm{NiH}$. It gave a physically meaningful model for the electronic structure of the $\mathrm{Ni}^{+}\left(3 \mathrm{~d}^{9}\right) \mathrm{H}^{-}$states by taking atomic orbital and spin angular momenta $\mathbf{L}$ and $\mathbf{S}$ of the $\mathrm{Ni}^{+}\left({ }^{2} D, d^{9}\right)$ to generate the off-diagonal matrix elements of a molecular 
Hamiltonian, where spin-orbit and rotational L- and $\mathbf{S}$-uncoupling terms introduce mixing between levels of the ${ }^{2} \Delta,{ }^{2} \Pi$ and ${ }^{2} \Sigma^{+}$states. The supermultiplet description explained the difficulties encountered in fitting infrared and far-infrared transitions in the $\mathrm{X}_{1}{ }^{2} \Delta_{5 / 2}$ and $\mathrm{X}_{2}{ }^{2} \Delta_{3 / 2}$ states of NiH and NiD with an effective Hamiltonian. Lipus et al. [32] reported that attempts to fit ${ }^{\mathrm{x}} \mathrm{NiH}$ or ${ }^{\mathrm{x}} \mathrm{NiD}$ $(\mathrm{x}=58,60,61,62)$ data with mass-scaled parameters had given unacceptably large standard deviations, and unphysically large Born-Oppenheimer breakdown corrections for H/D substitution; the authors attributed the limitations of their effective Hamiltonain to the influence of unobserved neighbouring levels.

Our purpose was to map out a larger set of energy levels for the low-lying states of NiD, using Fourier transform spectroscopy to resolve laser-induced fluorescence, and to assess the performance of the supermultiplet model when extended to higher energies, and/or with mixed isotopomer input. The original work by Gray et al [37] adjusted the parameters of a $15 \times 15$ energy matrix to represent ro-vibrational energies up to $v=2$ in each of the low-lying states of ${ }^{58} \mathrm{NiH}$, with uncertainties in the input energies varying from 0.05 to $1.5 \mathrm{~cm}^{-1}$. The model incorporated offdiagonal contributions between levels with $\Delta v=0$ and 1 . Subsequent experimental work on $\mathrm{NiH}$ $[38,39]$ has provided an extended set of energy levels for ${ }^{58,60,62} \mathrm{NiH}$, (up to $v=4$ for ${ }^{2} \Delta_{5 / 2}$ ) with $\sim 0.01$ $\mathrm{cm}^{-1}$ uncertainty, covering approximately the same energy range as our current work on NiD. This information provides a more stringent test for an expanded supermultiplet description of the lowlying energy levels. The supermultiplet energy matrix has been expanded (to $40 \times 40$ ), and now includes off-diagonal terms with $\Delta v \leq 2$, with some additional empirical parameters intended to compensate effects of more remote levels. This approach is successful for $\mathrm{NiH}$ in the region where all the vibronic states are observed. After that, fits converge with difficulty, and return increasingly unphysical values for some of the off-diagonal terms. By restricting the number of free parameters in the model (and accepting a larger root-mean-square error in the fit) we reproduce the ensemble of 
low-lying rovibronic energies in $\mathrm{NiH}$ and $\mathrm{NiD}$ reasonably well, although the $\Omega=3 / 2$ levels of $\mathrm{NiD}$ contribute significantly to the pool of 'outliers' in a multi-isotope fit. These are expected to be strongly affected by spin-orbit mixing with (unobserved) ${ }^{2} \Pi_{1 / 2}$ and ${ }^{2} \Sigma^{+}$, whose unperturbed positions are 'best estimate extrapolations' derived from $\mathrm{NiH}$ data.

\section{EXPERIMENT}

We produced ${ }^{58} \mathrm{NiD}$ in our DC sputter source [40] running with a $150 \mathrm{~mA}$ current between a copper loop anode and Ni cathode, with gas pressure around 1 Torr. The water-cooled cathode was drilled to allow a continuous flow of a commercial gas mixture $\left(5 \% \mathrm{D}_{2}\right.$ in Ar, from AirLiquide) through a vacuum chamber. The discharge products were probed by a cw single-mode ring dye laser operating around 17100 or $18100 \mathrm{~cm}^{-1}$ (with R6G or pyrromethene 567 dyes), with the laser beam intersecting the discharge plume about $1 \mathrm{~cm}$ below the anode. The dye laser was tuned to some of the stronger transitions (B ${ }^{2} \Delta_{5 / 2} \leftarrow \mathrm{X}{ }^{2} \Delta_{5 / 2}$ or $\mathrm{F}^{2} \Phi_{7 / 2} \leftarrow \mathrm{X}{ }^{2} \Delta_{5 / 2}$ ) transitions reported by Adakkai Kadavathu et al. in 1987. Backwards fluorescence was collected and transferred to a commercial FT spectrometer, passing through a $\lambda>590 \mathrm{~nm}$ filter to minimize laser scatter. The fluorescence was much weaker than had been seen (by eye) in $\mathrm{NiH}$ with the same set-up, and Fourier-transform resolved fluorescence spectra revealed that collisional energy transfer processes made a smaller contribution to the total fluorescence signal than in $\mathrm{NiH}$. They nevertheless populate the two levels of the $\mathrm{D} \Omega^{\prime}=3 / 2$ state (also reported by Adakkai Kadavathu and co-workers) and $v=$ 0 of the $\mathrm{E} \Omega^{\prime}=3 / 2$ state, from which fluorescence occurs to several levels of the two low-lying $\Omega^{\prime \prime}$ $=3 / 2$ states. Collisions following excitation of $\nu=1, \mathrm{~B}^{2} \Delta_{5 / 2}$ populate a second $\Omega^{\prime}=5 / 2$ level, that we assign as $v=1$ of the A state (weak transitions to $v=0$ were reported in ref.[25]). Tuning the laser to shorter wavelengths, collisions from $v=2, \mathrm{~B}^{2} \Delta_{5 / 2}$ populate another two $\Omega^{\prime}=3 / 2$ levels that we label $\mathrm{E}$ $\Omega^{\prime}=3 / 2, v=1$ and $\mathrm{I} \Omega^{\prime}=3 / 2, v=0$, scaling from $\mathrm{NiH}$. Figure 1 maps the observed bands whose term 
energies could be referenced to the ground electronic state. The direct fluorescence from $\mathrm{B}{ }^{2} \Delta_{5 / 2}$ to $\mathrm{X}{ }^{2} \Delta_{3 / 2}$ was very weak, but important in connecting the energy level stacks. Fluorescence from ${ }^{60} \mathrm{NiD}$ and ${ }^{62} \mathrm{NiD}$ was too weak to collect useable interferograms.

Figure 2 illustrates one of the dispersed fluorescence spectra of ${ }^{58} \mathrm{NiD}$, recorded at $0.04 \mathrm{~cm}^{-1}$ resolution (recording time $\sim 2$ hours). The laser excited the blend of $\mathrm{Q}_{\mathrm{ef}}(2.5)$ and $\mathrm{Q}_{\mathrm{fe}}(2.5) 1-0 \mathrm{~B}-\mathrm{X}$ transitions. Comparison with the spectrum pumped via the same transition in $\mathrm{NiH}$ (reproduced beneath) shows that collisions populate a quite different selection of excited states. The NiD spectrum is much weaker than $\mathrm{NiH}$, so higher gain settings were used to record the upper spectrum in Fig. 2. Argon atomic lines were more prominent in the NiD spectrum, and scatter from both the pump laser and the spectrometer's internal HeNe laser had to be reduced with optical filters. The high-pass glass filter placed outside the interferometer to remove dye-laser scatter also attenuates the rotational relaxation in the (1-0) B-X band of NiD. Scatter from the HeNe laser was reduced with a notch filter (NF633, Thorlabs) placed in front of the Si-avalanche detector, inside the spectrometer. Most of the B-X (1-1) band was also rejected by the notch filter. In both spectra, emissions following rotationally unselective electronic energy transfer contribute more to the total fluorescence signal than direct emission from the level populated by the laser.

\section{Analysis}

The direct fluorescence back to vibrational levels $0-5$ of the $\mathrm{X}_{1}{ }^{2} \Delta_{5 / 2}$ state, with extensive rotational relaxation, was readily assigned on all the ${ }^{58} \mathrm{NiD}$ spectra. Some of the collisionallyinduced bands above $14000 \mathrm{~cm}^{-1}$ had already been observed and assigned by Adakkai Kadavathu and co-workers[26], and combination differences could be used to confirm most of the excited state assignments for the bands seen at longer wavelength. Loomis-Wood plots were used to make 
rotational assignments in the regions where several bands overlap. Combination differences allowed us to reference nearly all the observed transitions to the lowest level of the $\mathrm{X}_{1}{ }^{2} \Delta_{5 / 2}$ state (see Figure 1), in a simple term-value fit (given in supplementary data).

Vibrational intervals were very regular for fluorescence series to the $\mathrm{X}_{1}{ }^{2} \Delta_{5 / 2}$ state, but vibrational/electronic assignments were less obvious for the $\Omega "=3 / 2$ states $\left(\mathrm{X}_{2}{ }^{2} \Delta_{3 / 2}\right.$ and $\left.\mathrm{W}_{1}{ }^{2} \Pi_{3 / 2}\right)$ in NiD. Guided by mass-scaling the parameters of a 'supermultiplet' fit for ${ }^{58} \mathrm{NiH}$, we identified the lower electronic states as depicted in Figure 3. The noteworthy features are the irregular vibrational separations, and unusually large e/f parity splittings in the $v^{\prime \prime}>0$ levels of the $\mathrm{X}_{2}{ }^{2} \Delta_{3 / 2}$ state. The parity splittings in $\mathrm{X}_{2}{ }^{2} \Delta_{3 / 2}$ are sometimes larger than in the other nearby $\Omega "=3 / 2$ state, nominally $\mathrm{W}_{1}{ }^{2} \Pi_{3 / 2}$. We identified some bands from three previously unreported upper state levels, assigning $\Omega^{\prime}$ on the basis of first lines in the branches observed. They are $v=1$ of the $A(\Omega=5 / 2)$ state (with transitions seen only to $\left.\mathrm{X}_{1}{ }^{2} \Delta_{5 / 2}\right), v=0$ of $\mathrm{I}(\Omega=3 / 2)\left(\mathrm{P}\right.$ and $\mathrm{R}$ branches to $\left.\mathrm{W}_{1}{ }^{2} \Pi_{3 / 2}\right)$ and $v=1$ of $\mathrm{E}(\Omega=3 / 2)$ (P and $\mathrm{R}$ branches to only $v=0$ of $\left.\mathrm{X}_{2}{ }^{2} \Delta_{3 / 2}\right)$. Table 1 lists the effective band constants for these excited levels, obtained by fitting the output from the term energy fit to an empirical formula $T_{v, J, \Omega, e / f}=T_{v}+\sum_{m=1,3} Y_{0, m}\left[\left[J(J+1)-\Omega^{2}\right]\right]^{m} \pm \frac{1}{2}\left(q_{v}[J(J+1)]^{\Omega}+q_{D}[J(J+1)]^{2 \Omega}\right)$.

Levels with e parity take the positive splitting $\mathrm{q}_{\mathrm{v}}\left[(J(J+1)]^{\Omega}+\ldots\right.$ terms in eq $(1)$, and $\mathrm{f}$ parity levels take the negative one. The same approach for the term energies of $\mathrm{X}_{1}{ }^{2} \Delta_{5 / 2}, \mathrm{X}_{2}{ }^{2} \Delta_{3 / 2}$ and $\mathrm{W}_{1}{ }^{2} \Pi_{3 / 2}$ states produces the effective band parameters listed in Table 2, where the vibrational intervals and distortion constants for the $\mathrm{X}_{2}{ }^{2} \Delta_{3 / 2}$ state underline the inadequacy of this simplistic model. These band parameters give a convenient representation for the observed bands, but have no predictive powers for higher vibrational levels. The supermultiplet approach should allow empirical parameters to be replaced with more physically meaningful ones, offset by a more complicated Hamiltonian. However, there is a serious impediment to this approach for ${ }^{58} \mathrm{NiD}$. None of the 
spectra we have recorded so far has produced fluorescence allowing the low-lying $\Omega=1 / 2$ states $\left({ }^{2} \Sigma^{+}\right.$ and ${ }^{2} \Pi_{1 / 2}$ ) to be located with respect to the electronic ground state. An isolated branch (around $14800 \mathrm{~cm}^{-1}$ ) with no resolved parity-splittings is strongly reminiscent of some $\Delta \Omega=0$ transitions between $\Omega=1 / 2$ states seen in $\mathrm{NiH}$ spectra [38], but we have no upper state combination differences to link these lines to the assigned systems. To compensate the gap in information, we opted for a multi-isotope deperturbation analysis, fitting ro-vibrational term values up to $6500 \mathrm{~cm}^{-1}$ for the three strongly-interacting low-lying electronic states $\left({ }^{2} \Delta,{ }^{2} \Pi\right.$ and $\left.{ }^{2} \Sigma^{+}\right)$for ${ }^{\mathrm{x}} \mathrm{NiH}$ and NiD. ${ }^{58} \mathrm{NiD}$ term energies came from this analysis. Energy level data for ${ }^{\mathrm{x}} \mathrm{NiH}(\mathrm{x}=58,60$ and 62$)$ were taken from earlier work from the Lyon group [38,39].

\section{Supermultiplet Hamiltonian : extension and modification.}

The effective parameters of Tables 1 and 2 give a crude but convenient model for recognizing the lines seen in the spectra. Aiming to produce a model with better predictive powers for the lowest electronic states, we proceeded to fit the low-lying energy levels in $\mathrm{NiH}$ and $\mathrm{NiD}$ in an slightly modified version of the 'supermultiplet' model used by Gray and co-workers [26]. The challenge was to extend this physically reasonable model to higher vibrational levels (we observe up to $v=5$ in the $\mathrm{X}_{1}{ }^{2} \Delta_{5 / 2}$ state of NiD, but fewer levels of the other states, see table 4), and to use appropriate isotopic scaling of parameters (defined for ${ }^{58} \mathrm{NiH}$ ) so that they reproduce measured energy levels of other isotopic forms $\left({ }^{60,62} \mathrm{NiH},{ }^{58} \mathrm{NiD}\right)$.

We chose to take Brown's $\boldsymbol{N}^{2}$-effective Hamiltonian [41-43] for the 'unperturbed' basis states, for which vibrational, rotational, spin-orbit, spin rotation and $\Lambda$-doubling parameters were represented by simple Dunham-type parameters, $x_{v}=x_{e}+\sum_{k+1} x_{k}\left(v+\frac{1}{2}\right)^{k}$.

The $\mathrm{X}^{2} \Delta, \mathrm{W}^{2} \Pi$ and $\mathrm{V}^{2} \Sigma^{+}$electronic basis states were thus represented by 


$$
\begin{aligned}
& H_{e f f}=T_{v}+G_{v}+B_{v} \mathbf{N}^{2}-D_{v} \mathbf{N}^{4}+\gamma_{v} \mathbf{N} . \mathbf{S}+\gamma_{D, v} \mathbf{N}^{2}(\mathbf{N} . \mathbf{S})+A_{v} \mathbf{L} . \mathbf{S} \\
& +\frac{1}{2}\left[\left(p_{v}^{\Pi}+q_{v}^{\Pi}\right)\left(S_{+}^{2}+S_{-}^{2}\right)-\left(p_{v}^{\Pi}+2 q_{v}^{\Pi}\right)\left(J_{+} S_{+}+J_{-} S_{-}\right)+q_{v}^{\Pi}\left(J_{+}^{2}+J_{-}^{2}\right)-p_{v}^{\Delta}\left(J_{+}^{3} S_{+}+J_{-}^{3} S_{-}\right)+q_{v}^{\Delta}\left(J_{+}^{4}+J_{-}^{4}\right)\right]
\end{aligned}
$$

The spin-orbit parameter $A$ was assumed to be isotopically invariant. When it could not be determined from experimental data, it was constrained to the free $\mathrm{Ni}^{+}$ion value $\left(-603 \mathrm{~cm}^{-1}\right)$. Vibrational dependence could be determined only for the $\mathrm{X}^{2} \Delta$ state in $\mathrm{NiH}$, so simple mass scaling $A_{v}^{(\alpha)}=A_{e}^{(1)} \sqrt{\left(\frac{\mu_{(1)}}{\mu_{(\alpha)}}\right)}\left(v+\frac{1}{2}\right)$ provided an initial value for $A_{1}$ in NiD.

We found that the 'supermultiplet' model struggled to reproduce the lowest $\mathrm{NiH}$ energy levels to their estimated experimental uncertainty, and interpreted this as the consequence of truncation of the off-diagonal terms at $\Delta v \leq 2$. Introducing empirical spin-rotation $(\gamma)$ and $\Lambda$-doubling $(p$ and $q)$ parameters in eq. (3) compensated these problems in the lowest vibrational levels. As they are accounting only for effects of remote states, these terms were expected to be small. They were assumed to scale isotopically according to their dependences on $B_{\mathrm{v}}$ (given in refs $[28,44]$ );

$$
\begin{aligned}
& p_{v}^{\Pi}=\frac{4 \zeta_{3 d} B_{v}}{E_{\Pi, v}-E_{\Sigma, v^{\prime}}}, \\
& q_{v}^{\Pi}=\frac{4 B_{v}^{2}}{E_{\Pi, v}-E_{\Sigma, v^{\prime}}}, \\
& p_{v}^{\Delta}=\frac{96 \zeta_{3 d} B_{v}^{3}}{\left(E_{\Delta, v}-E_{\Sigma, v^{\prime}}\right)\left(E_{\Delta, v}-E_{\Pi, v^{\prime}}\right)^{2}} \text { and } \\
& q_{v}^{\Delta}=\frac{48 B_{v}^{4}}{\left(E_{\Delta, v}-E_{\Sigma, v^{\prime}}\right)\left(E_{\Delta, v}-E_{\Pi, v^{\prime}}\right)^{2}} .
\end{aligned}
$$


Having chosen to work with the $\mathbf{N}^{2}$ Hamiltonian, we had to incorporate the difference in electronic term energies associated with the $\mathbf{R}^{\mathbf{2}}$ and $\mathbf{N}^{\mathbf{2}}$ Hamiltonians when using ${ }^{58} \mathrm{NiH}$ parameters to calculate energies of isotopically substituted forms. The difference between electronic energy origins for the different ${ }^{2} \Lambda$ states when expressed in $\mathbf{R}^{2}$ or $\mathbf{N}^{2}$ forms is

$$
\left\langle{ }^{2 S+1} \Lambda\left|B(R)\left(\mathbf{R}^{2}-\mathbf{N}^{2}\right)\right|^{2 S+1} \Lambda\right\rangle=B_{\Lambda}(R)\left[L(L+1)-2 \Lambda^{2}\right]
$$

where $B_{\Lambda}(R)$ is rotational constant of the ${ }^{2} \Lambda$ state, and $L=2$ (associated with the $\mathrm{Ni}^{+} 3 \mathrm{~d}^{9}$ configuration) Setting $\mathrm{T}_{\Lambda}\left(\mathbf{R}^{2}\right)+\mathrm{B}_{\Lambda} \mathbf{R}^{2}$ equal to $\mathrm{T}_{\Lambda}\left(\mathbf{N}^{2}\right)+\mathrm{B}_{\Lambda} \mathbf{N}^{2}$, we obtained the following relations:

$T_{\Delta}\left(\mathbf{N}^{2}\right)-T_{\Delta}\left(\mathbf{R}^{2}\right)=-2 B_{\Delta}$

$T_{\Pi}\left(\mathbf{N}^{2}\right)-T_{\Pi}\left(\mathbf{R}^{2}\right)=-2 B_{\Pi}$

$T_{\Sigma}\left(\mathbf{N}^{2}\right)-T_{\Sigma}\left(\mathbf{R}^{2}\right)=(0.86)^{2} \times 6 B_{\Sigma}$

$T_{\mathrm{e}}$ for the ${ }^{2} \Delta$ state is taken to be zero in both $\mathbf{R}^{2}$ and $\mathbf{N}^{2}$ models, so the energy shifts for equilibrium term energies $T_{\mathrm{e}}$ of the ${ }^{2} \Pi$ and ${ }^{2} \Sigma^{+}$states become:

$T_{e, \Pi}\left(\mathbf{N}^{2}\right)-T_{e, \Pi}\left(\mathbf{R}^{2}\right)=4 B_{e, \Pi}+2 B_{e, \Delta}$

$T_{e, \Sigma}\left(\mathbf{N}^{2}\right)-T_{e, \Sigma}\left(\mathbf{R}^{2}\right)=(0.86)^{2} \times 6 B_{e, \Sigma}+2 B_{e, \Delta}$

$T_{v, \Lambda} \quad B_{v, \Lambda}$ etc were calculated for isotopically substituted forms according to Le Roy's formulation[45] from Dunham-type parameters for each state,

$Y_{l, m}^{(\alpha)}=\left[Y_{l, m}^{(1)}+\frac{\Delta M_{H}^{(\alpha)}}{M_{H}^{(\alpha)}} \delta_{l, m}^{H}+\frac{\Delta M_{N i}^{(\alpha)}}{M_{N i}^{(\alpha)}} \delta_{l, m}^{N i}\right]\left(\frac{\mu_{1}}{\mu_{\alpha}}\right)^{m+l / 2}$ 
where $\delta_{l, m}^{H}$ and $\delta_{l, m}^{N i}$ are Born-Oppenheimer breakdown correction parameters; $\Delta M_{X}^{(\alpha)}=M_{X}^{(\alpha)}-M_{X}^{(1)}, M_{H}^{(x)}$ and $M_{N i}^{(x)}$ represent the atomic masses of the $H$ and $N i$ atoms, and $\mu$ the reduced masses.

Brown and Watson [46] have given analogous reduced-mass scaling relationships for the spinrotation terms, $\gamma_{l, m}^{(\alpha)}=\gamma_{l, m}^{(1)}\left(\frac{\mu^{(1)}}{\mu^{(\alpha)}}\right)^{1+m+\frac{l}{2}}$

For the $\Lambda$-doubling parameters, we take :

$$
\begin{aligned}
& p_{l, m}^{(\alpha)}(\Pi)=p_{l, m}^{(1)}(\Pi)\left(\frac{\mu_{1}}{\mu_{\alpha}}\right)^{1+m+l / 2}, \\
& q_{l, m}^{(\alpha)}(\Pi)=q_{l, m}^{(1)}(\Pi)\left(\frac{\mu_{1}}{\mu_{\alpha}}\right)^{2+m+l / 2}, \\
& p_{l, m}^{(\alpha)}(\Delta)=p_{l, m}^{(1)}(\Delta)\left(\frac{\mu_{1}}{\mu_{\alpha}}\right)^{3+m+l / 2} \text { and } \\
& q_{l, m}^{(\alpha)}(\Delta)=q_{l, m}^{(1)}(\Delta)\left(\frac{\mu_{1}}{\mu_{\alpha}}\right)^{4+m+l / 2}
\end{aligned}
$$

Although they were expected to be significant for hydrogen/deuterium substitution, the leading Born-Oppenheimer breakdown parameters $\delta_{00}^{H}, \delta_{10}^{H}$ could not be determined in a statistical sense from the data available, because they are strongly correlated with vibrational off-diagonal parameters $f_{\Lambda \Lambda^{\prime}}$. We used eq. (7) to calculate the shifts in energy origins for the ${ }^{2} \Lambda$ electronic states of NiD relative to those of $\mathrm{NiH}$ based on the differences in $\mathbf{R}^{2}$ and $\mathbf{N}^{2}$ Hamiltonians (eq. 4), and 
then held these (effective) $\delta_{0,0}^{H}$ parameters fixed in the fit. The resulting expressions, and their numerical values, are :

$$
\begin{aligned}
& T_{e, \Lambda}(N i D)-T_{e, \Lambda}(N i H)=\left(\frac{M^{D}-M^{H}}{M^{H}}\right) \delta_{00}^{H}(\Lambda) \\
& \delta_{02}^{H}(\Delta)=-0.9\left(\frac{M^{D}}{M^{D}-M^{H}}\right) \times\left[4 B_{e(\Pi)}+2 B_{e(\Delta)}\right] \times\left[\left(\frac{\mu_{N i H}}{\mu_{N i D}}\right)-1\right] \approx-45 \mathrm{~cm}^{-1}, \\
& \left\langle{ }^{2} \Delta_{3 / 2}\left|\hat{\mathbf{H}}_{\mathrm{rot}}+\hat{\mathbf{H}}_{\mathrm{so}}\right|{ }^{2} \Pi_{3 / 2}\right\rangle=-603 f_{v_{\Delta}, v_{\Pi}}+2 B_{v_{\Delta}, v_{\Pi}} \approx-49 \mathrm{~cm}^{-1} .
\end{aligned}
$$

Nickel isotope substitution was treated in the same way, constraining the values $\delta_{00}^{N i}(\Pi)=-0.8$ and $\delta_{00}^{N i}(\Sigma)=-0.9 \mathrm{~cm}^{-1}$ in the multi-isotope fits. The $\delta_{01}^{H}$ parameters were strongly correlated with the off-diagonal $B_{\mathrm{vv}^{\prime}}$ parameters, and were set to zero. Only one Born-Oppenheimer breakdown parameter was finally optimized in our fits : $\delta_{02}^{H}(\Delta)=-4.07(14) \times 10^{-5} \mathrm{~cm}^{-1}$, for the ${ }^{2} \Delta$ state. It gave a small improvement to the overall root-mean-square deviation of the fit by reducing obs-calc deviations for the higher $J$ levels in $v=0$ of the ${ }^{2} \Delta$ state of NiD.

Off-diagonal spin-orbit mixing terms have been taken from the $\boldsymbol{R}^{2}$ supermultiplet model detailed by Gray et al. These included spin-orbit coupling terms producing the strong mixing between ${ }^{2} \Delta_{3 / 2}$ and ${ }^{2} \Pi_{3 / 2}$ states and between ${ }^{2} \Pi_{1 / 2}$ and ${ }^{2} \Sigma^{+}$states, originating from $\zeta_{\mathrm{Ni}+3 d}\left(-603 \mathrm{~cm}^{-1}\right)$. The parameters $A_{\Pi \Delta}$ and $A_{\Sigma \Pi}$ are scaled by a vibrational overlap factor, $f_{\Pi \Delta}$ (or $f_{\Sigma \Pi}$ ), largest for $\Delta v=0$ but nonzero elsewhere. Gray's additional scaling coefficient $\mathrm{c}_{\Sigma \Pi}$ of 0.86 , justified by indications from ab initio work that the low-lying ${ }^{2} \Sigma^{+}$state has around $25 \% \mathrm{Ni} 3 \mathrm{~d}^{10}$ character, was applied to all ${ }^{2} \Pi \sim{ }^{2} \Sigma^{+}$ matrix elements (eqs. 11, 12). The vibrational overlap integrals $f_{v_{\Lambda^{\prime}}, v_{\Lambda^{\prime}}}=\left\langle v_{\Lambda}, \mid v_{\Lambda^{\prime},}\right\rangle$, and rotationally- 
dependent terms, $B_{v_{\Lambda^{\prime}, \nu^{\prime}}}=\frac{\hbar^{2}}{2 \mu}\left\langle v_{\Lambda}\left|\frac{1}{r^{2}}\right| v_{\Lambda^{\prime}}\right\rangle$, were calculated using Le Roy's program LEVEL[47], taking as input the RKR potentials calculated [48] from Dunham-type representations of $G_{\mathrm{v}}$ and $B_{\mathrm{v}}$ (eq. (2)).

Matrix elements coupling the ${ }^{2} \Delta,{ }^{2} \Pi$ and ${ }^{2} \Sigma^{+}$states are then:

$$
\begin{aligned}
& \left\langle{ }^{2} \Delta_{5 / 2}\left|\hat{\mathbf{H}}_{\mathrm{rot}}+\hat{\mathbf{H}}_{\mathrm{SO}}\right|{ }^{2} \Pi_{3 / 2}\right\rangle=-2 B_{v_{\Delta}, v_{\Pi}} \times \sqrt{(J+5 / 2)(J-3 / 2)} \\
& \left\langle{ }^{2} \Delta_{3 / 2}\left|\hat{\mathbf{H}}_{\mathrm{rot}}+\hat{\mathbf{H}}_{\mathrm{SO}}\right|{ }^{2} \Pi_{3 / 2}\right\rangle=-603 f_{v_{\Delta}, v_{\Pi}}+2 B_{v_{\Delta}, v_{\Pi}} \\
& \Delta E_{\text {Spin-rotation }}^{f}=0.5\left(\gamma+\gamma_{D} x\right)(N+1) \\
& \left\langle{ }^{2} \Pi_{3 / 2}\left|\hat{\mathbf{H}}_{\mathrm{rot}}+\hat{\mathbf{H}}_{\mathrm{so}}\right|{ }^{2} \Sigma_{1 / 2}^{+}\right\rangle=-0.86 \times \sqrt{6} B_{v_{\Pi}, v_{\Sigma}} \times \sqrt{(J-1 / 2)(J+3 / 2)} \\
& \left\langle{ }^{2} \Pi_{1 / 2}\left|\hat{\mathbf{H}}_{\text {rot }}+\hat{\mathbf{H}}_{\mathrm{so}}\right|{ }^{2} \Sigma_{1 / 2}^{+}\right\rangle=\frac{1}{2}\left[-603 \times 0.86 \times \sqrt{6} f_{v_{\Pi}, v_{\Sigma}}\right]+0.86 \times \sqrt{6} B_{v_{\Pi}, v_{\Sigma}} \times[1 \mp(J+1 / 2)],
\end{aligned}
$$

where upper/lower sign applies to levels of elf parity. The matrix elements are given in Table 4.

The Dunham-type (rounded) parameters returned from the non-linear least-squares fit (subroutine NLLSSRR.f from Le Roy's DParFit program [49]) are listed in Table 5. The vibrational overlap integrals needed in the off-diagonal terms are given in Table 6a, and the off-diagonal rotational terms in Table $6 b$.

Given the unbalanced data set, with significantly less information on the ${ }^{2} \Sigma^{+}$and ${ }^{2} \Pi_{1 / 2}$ states of NiH (and none at all for NiD) than for ${ }^{2} \Pi_{3 / 2},{ }^{2} \Delta_{3 / 2}$ and ${ }^{2} \Delta_{5 / 2}$ states, it was not particularly surprising to find that 'supermultiplet' multi-isotope fits failed to converge to a completely convincing solution. A weighted fit (according to $\mathrm{w}=1 /\left(\sigma_{\text {exp }}\right)^{2}$ ) returned unrealistic values of many parameters, without 
reproducing the energy terms within quoted uncertainties. This led us to introduce some of the constraints detailed above, and to reduce the weights of levels known to be in strong interaction with unobserved $\Omega^{\prime \prime}=1 / 2$ states for which we have less confidence in the predicted energies. The parameters given in Table 5 are thus not a unique solution to this problem, but represent our best compromise retaining a realistic model with only the leading parameters optimized. The overall weighted root-mean-square deviation of the fit is 3, (with unweighted root-mean-square deviation $0.5 \mathrm{~cm}^{-1}$ ), as illustrated in Figure 3, but the model should give reasonable predictions for higherlying levels. These are given as supplementary data.

\section{Conclusion}

We believe that this model offers the best predictions currently available for unobserved levels of different isotopic forms of $\mathrm{NiH}$, although the predictions for rovibrational levels of the low $\Omega\left({ }^{2} \Pi_{1 / 2}\right.$ and $\left.{ }^{2} \Sigma^{+}\right)$states are not particularly secure, since few levels were actually observed and included in our model, and those few were for ${ }^{58} \mathrm{NiH}$ alone. For NiD, $v=0$ of the ${ }^{2} \Sigma^{+}$state is predicted to lie $\sim 2080 \mathrm{~cm}^{-1}$ above the lowest level of the ${ }^{2} \Delta_{5 / 2}$ ground state. The level is sufficiently remote from others that it should be unambiguously assignable in resolved fluorescence, provided that an excitation (probably to $a^{2} \Pi_{3 / 2}$ state) can be found with significant transition dipole moments both to ${ }^{2} \Sigma^{+}$and any of the low-lying $\Omega=3 / 2$ or $5 / 2$ levels reported here. The situation is more delicate for higher vibrational levels, where our model places rovibrational levels $J=2.5$ e parity for ${ }^{2} \Sigma^{+} v=1$ at $3363,{ }^{2} \Pi_{1 / 2} v=0$ at 3555 and ${ }^{2} \Delta_{3 / 2} v=2$ at $3602 \mathrm{~cm}^{-1}$ (none of them being 'pure' states). The separations are even smaller for $\mathrm{f}$ parity levels, where the model returns ${ }^{2} \Sigma^{+} v=1$ at 3409 and ${ }^{2} \Pi_{1 / 2} v=0$ at $3530 \mathrm{~cm}^{-1}$. Even small changes in the parameters result in shifts easily of the order of the rotational constants in NiD, so assignment is likely to be more difficult, particularly if, as with $\mathrm{NiH}$, only one branch of a given transition to ${ }^{2} \Pi_{1 / 2}$ appears strongly 
in the spectrum. Figure 4 illustrates the predictions for $J=2.5$ levels of NiD from this model, and those from the ab initio work of ref. [12]. The figure shows that the ab initio calculations actually match remarkably well $\left(\mid\right.$ obs-calc $\left.\mid<50 \mathrm{~cm}^{-1}\right)$ for both components of the $\mathrm{X}^{2} \Delta$ state. The differences between $a b$ initio and supermultiplet model predictions are of the order of $+300 \mathrm{~cm}^{-1}$ for the ${ }^{2} \Sigma^{+},{ }^{2} \Pi_{1 / 2}$ and ${ }^{2} \Pi_{3 / 2}$ levels, but respect the same ordering of states (the supermultiplet fit places the ${ }^{2} \Pi_{3 / 2}$ levels within $2 \mathrm{~cm}^{-1}$ of observation). The main difference between the two models is that the ab initio work included a modest increase in the $<{ }^{2} \Sigma^{+}\left|\mathrm{H}_{\mathrm{SO}}\right|^{2} \Pi_{1 / 2}>$ spin-orbit matrix elements as a function of internuclear distance, where our supermultiplet fit imposed a constant value.

Spectroscopic data at higher temperatures are required for example in the modeling of cool stellar atmospheres. On the basis of abundances generated by nucleosysnthesis, one might expect $\mathrm{NiH}$ to be observed in cool stars: $\mathrm{Ni}$ is second only to $\mathrm{Fe}$ in the transition metal abundances. Lambert and Mallia's work [50] in the early '70s found plausible coincidences with the 1930's reports of $\mathrm{NiH}$ emission [20,21], implying that $\mathrm{NiH}$ might become a molecular tracer in stellar spectra. But a later wave of interest in supplying reference transition data for $\mathrm{NiH}$, notably O'Brien and co-workers using laser excitation cavity-enhanced spectroscopy [51-53], and in resolved fluorescence in Lyon [36,38,40] gave no confirmation, and our comparisons between more recent laboratory spectra and the extensive sunspot atlas now available at the Kitt Peak archive (ftp://nsokp.nso.edu/pub/atlas/) [54] suggest that the matches reported in ref [50] were accidental coincidences with very weak or blended features.

Representing energy levels of the strongly-interacting electronic states of $\mathrm{NiH}$ to experimental accuracy remains a spectroscopic challenge. A model taking analytical potential curves and radial spin-orbit functions rather than Dunham-type parameters should ultimately 
provide a more compact set of parameters, and be better suited for extrapolation to the higher rotational levels expected to dominate in stellar spectra. Tools to do this exist. The program DUO from Yurchenko et al. [55], for example, has been used to predict hot spectra for many species [56]. Coupled-channel representations based on $\mathrm{V}(\mathrm{R})$ functions [57] have been very successful in describing spin-orbit perturbations between pairs of triplet and singlet states in heavy alkali dimers. Work on $\mathrm{Rb}_{2}$ [58] and $\mathrm{KRb}$ [59] give just two illustrations of reduction of large data sets with the approaches of Bergeman and Stolyarov, including isotopically substituted species. But more information is needed on the heavily mixed ${ }^{2} \Sigma^{+}$and ${ }^{2} \Pi_{1 / 2}$ states of NiD before we can implement this approach sensibly for $\mathrm{NiH}$, underlining the need for further experimental work to access these states. We believe that the present analysis is sufficiently robust for $\mathrm{NiD}$ (and $\mathrm{NiH}$ ) to give proper predictions as to where we should be looking for them.

\section{Acknowledgements}

We gratefully acknowledge financial support from the 'Programme National de Physique Stellaire' (PNPS 2016) CNRS/INSU, CEA and CNES, France. 
Table 1. Effective band constants for $v=1 \mathrm{~A}(\Omega=5 / 2), v=1$ of $\mathrm{E}(\Omega=3 / 2)$ and $v=0$ of $\mathrm{I}(\Omega=3 / 2)$ identified in collisionally induced fluorescence of NiD. Values in $\mathrm{cm}^{-1}$, with $1 \sigma$ deviations in parenthesis, in units of last digit. Energies established with respect to the first ground state level, $v=0, J=2.5 \mathrm{X}^{2} \Delta_{5 / 2}$. The parity components of the A $\Omega=5 / 2 v=1$ level, resolved only above $J=7.5$, are not assigned, so the choice of sign for $q_{v}$ is arbitrary. An avoided crossing around $J=10 \mathrm{e}$ leads to the large RMS deviation for this level.

\begin{tabular}{lccc}
\hline Parameter & $\mathrm{A}(\Omega=5 / 2) v=1$ & $\mathrm{I}(\Omega=3 / 2) v=0$ & $\mathrm{E}(\Omega=3 / 2) v=1$ \\
\hline$T_{v}$ & $16657.09(2)$ & $17362.58(1)$ & $17503.54(2)$ \\
$B_{v}$ & $3.0682(9)$ & $3.1518(5)$ & $3.04265(101)$ \\
$10^{4} D_{v}$ & $1.988(116)$ & $1.216(39)$ & $3.21(16)$ \\
$10^{7} H_{v}$ & $-6.56(40)$ & & $5.35(68)$ \\
$q_{v}$ & $1.17(12) \times 10^{-6}$ & $-8.24(18) \times 10^{-4}$ & $1.290(5) \times 10^{-2}$ \\
$q_{D}$ & & & $-7.45(35) \times 10^{-7}$ \\
RMS deviation & 0.041 & 0.008 & 0.046 \\
$J_{\max }$ & 13.5 & 11.5 & 13.5 \\
\hline
\end{tabular}


Table 2: Band constants for the low-lying states of NiD determined from fit of term values to expression (1). Parameters in $\mathrm{cm}^{-1}$, with $1 \sigma$ deviations indicated in parenthesis in units of last digit. Note that e parity levels only were available for $v=3$ of $\mathrm{X}_{2}{ }^{2} \Delta_{3 / 2}$.

\begin{tabular}{|c|c|c|c|c|c|c|c|}
\hline State & $\mathrm{v}$ & $G_{v}$ & $B_{v}$ & $10^{4} D_{v}$ & $10^{3} q_{v}$ & $10^{8} q_{D}$ & RMS dev. \\
\hline \multirow[t]{6}{*}{$\mathrm{X}_{1}^{2} \Delta_{5 / 2}$} & 0 & 0.000 & $3.9913(6)$ & $1.299(19)$ & & & 0.004 \\
\hline & 1 & $1390.156(52)$ & $3.9012(8)$ & $1.292(33)$ & & & 0.004 \\
\hline & 2 & $2742.422(50)$ & $3.8110(6)$ & $1.272(19)$ & & & 0.005 \\
\hline & 3 & $4057.041(51)$ & $3.7217(7)$ & $1.265(25)$ & & & 0.003 \\
\hline & 4 & $5334.214(56)$ & $3.6326(9)$ & $1.251(32)$ & & & 0.008 \\
\hline & 5 & $6574.095(56)$ & $3.5439(14)$ & $1.246(88)$ & & & 0.002 \\
\hline \multirow[t]{4}{*}{$\mathrm{X}_{2}{ }^{2} \Delta_{3 / 2}$} & 0 & $977.739(43)$ & $3.9967(5)$ & $1.475(20)$ & $-0.888(16)$ & & 0.013 \\
\hline & 1 & $2296.313(44)$ & $3.9574(6)$ & $0.879(32)$ & $-6.049(32)$ & & 0.024 \\
\hline & 2 & $3586.312(46)$ & $3.8982(11)$ & $-1.593(79)$ & $-8.25(61)$ & & 0.177 \\
\hline & 3 & $4841.961(57)$ & $3.8139(20)$ & $3.621(170)$ & - & & 0.120 \\
\hline \multirow[t]{2}{*}{$\mathrm{W}_{1}^{2} \Pi_{3 / 2}$} & 0 & $2806.840(44)$ & $3.8553(6)$ & $1.346(29)$ & $-15.0(57)$ & $-4.4(21)$ & 0.013 \\
\hline & 1 & $4175.053(46)$ & $3.7556(10)$ & $1.532(65)$ & $-15.5(33)$ & & 0.019 \\
\hline
\end{tabular}


Table 3

\begin{tabular}{|c|c|c|c|c|c|}
\hline & ${ }^{2} \Delta_{5 / 2}$ & ${ }^{2} \Delta_{3 / 2}$ & ${ }^{2} \Pi_{3 / 2}$ & ${ }^{2} \Pi_{1 / 2}$ & ${ }^{2} \Sigma_{1 / 2}^{+}$ \\
\hline${ }^{2} \Delta_{5 / 2}$ & $\begin{array}{l}A+0.5 \gamma+\gamma_{D} \\
+B(z-2)-D z(z-3) \\
\pm 0.25 q_{D}(z-1)(z-4)(J+0.5)\end{array}$ & $-\sqrt{(z-4)}\left[\begin{array}{l}(B-0.5 \gamma) \\
-0.5 \gamma_{D}(z+1)-2 D z \\
\mp 0.25(z-1)(J+0.5) \times \\
\left(2 q+p_{D}-q_{D}(z+2)\right)\end{array}\right]$ & $\begin{array}{l}-2 B_{v_{\Delta}, v_{\Pi}} \times \\
\sqrt{(J+2.5)(J-1.5)}\end{array}$ & 0 & 0 \\
\hline${ }^{2} \Delta_{3 / 2}$ & symmetric & $\begin{array}{l}-A-1.5 \gamma-\gamma_{D}(2 z+1) \\
+B(z+2)-D z(z+5) \\
\mp 0.5(z-1)(J+0.5) \times \\
{\left[p+4 q+p_{D}(z+2)-0.5 q_{D}(5 z+4)\right]}\end{array}$ & $-603 f_{v_{\Delta}, v_{\Pi}}+2 \mathrm{~B}_{v_{\Delta}, v_{\Pi}}$ & $\begin{array}{l}-2 B_{v_{\Delta}, v_{\Pi}} \times \\
\sqrt{(J-0.5)(J+1.5)}\end{array}$ & 0 \\
\hline${ }^{2} \Pi_{3 / 2}$ & symmetric & symmetric & $0.5 A+y\left[\begin{array}{l}B-D(y+1) \\
-0.5 \gamma_{D} \mp 0.5 q_{D}(J+0.5)\end{array}\right]$ & $\sqrt{y}\left[\begin{array}{l}-B+2 D(y+1) \\
+0.5\left(\gamma+\gamma_{D}(y+2)\right) \\
\pm 0.5(J+0.5)\left(0.5 p_{D}+q+q_{D}(y+2)\right)\end{array}\right]$ & $\begin{array}{l}-2.1066 B_{v_{\Pi}, v_{\Sigma}} \times \\
\sqrt{(J-0.5)(J+1.5)}\end{array}$ \\
\hline${ }^{2} \Pi_{1 / 2}$ & symmetric & symmetric & symmetric & $\begin{array}{l}-0.5 A+B(y+2)-\gamma \\
-D(y+1)(y+4) \\
-0.5 \gamma_{D}(3 y+4) \mp 0.5(J+0.5) \times \\
{\left[p+p_{D}(y+2)+2 q+q_{D}(3 y+4)\right]}\end{array}$ & $\begin{array}{l}-635 f_{v_{\Pi}, v_{\Sigma}}+ \\
2.1066 B_{v_{\Pi}, v_{\Sigma}}[1 \mp(J+0.5)]\end{array}$ \\
\hline & symmetric & symmetric & symmetric & symmetric & $B x-D x^{2}+\Delta E_{\text {Spin-Rotation }}^{(b)}$ \\
\hline
\end{tabular}


Caption to Table 3. Matrix elements of the $\mathbf{N}^{2}$ Hamiltonian for the ${ }^{2} \Delta,{ }^{2} \Pi$ and ${ }^{2} \Sigma^{+}$states and $\mathbf{R}^{2}$ Hamiltonian for the ${ }^{2} \Delta \sim \sim^{2} \Pi \sim{ }^{2} \Sigma^{+}$ interactions. The upper and lower signs refer to $e$ and $f$ parities, respectively. ${ }^{(a)}$ This block repeats for $v=0-5$, with off-diagonal elements assumed to be zero for interactions with $\Delta v>2$.

Footnote to table 3 .

(a) $z=(J+0.5)^{2}, y=z-1$ and $x=N(N+1)$.

(b) $\Delta E_{\text {Spin-rotation }}^{e}=0.5\left(\gamma+\gamma_{D} X\right)(N)$ and $\Delta E_{\text {Spin-rotation }}^{f}=0.5\left(\gamma+\gamma_{D} X\right)(N+1)$ 
Table 4. The range of observed vibrational levels, and associated ranges of term ${ }^{\mathrm{x}} \mathrm{NiH}$ and ${ }^{58} \mathrm{NiD}$. The complete list of term energies is available as supplementary

\begin{tabular}{ccccc}
\hline State & $\begin{array}{c}\text { Obs } \\
\text { (range) }\end{array}$ & ${ }^{58} \mathrm{NiH}$ & ${ }^{60} \mathrm{NiH}$ & ${ }^{62} \mathrm{NiH}$ \\
\hline $\mathrm{X}^{2} \Delta_{5 / 2}$ & $v$ & $0-4$ & $0-4$ & $0-1$ \\
& $\mathrm{~T}_{v J}$ & $0-7740$ & $0-7740$ & $0-4170$ \\
$\mathrm{X}^{2} \Delta_{3 / 2}$ & $v$ & $0-2$ & $0-1$ & $0-1$ \\
& $\mathrm{~T}_{v J}$ & $973-5730$ & $973-5325$ & $972-5125$ \\
$\mathrm{~W}^{2} \Pi_{3 / 2}$ & $v$ & $0-2$ & $0-2$ & 0 \\
& $\mathrm{~T}_{v J}$ & $2610-6525$ & $2610-6522$ & $2609-4713$ \\
$\mathrm{~W}^{2} \Pi_{1 / 2}$ & $v$ & $0-1$ & & \\
$\mathrm{~V}^{2} \Sigma^{+}$ & $\mathrm{T}_{v J}$ & $3454-5680^{(\mathrm{a})}$ & & \\
\hline
\end{tabular}

(a) $e$ parity data only for $v=1$ 
Table 5

Parameters (in $\mathrm{cm}^{-1}$ ) describing the basis states ${ }^{2} \Delta,{ }^{2} \Pi$ and $2 \Sigma^{+}$for ${ }^{58} \mathrm{NiH}$ from multi-isotope fit, with scaled parameters for ${ }^{58} \mathrm{NiD}$. $1 \sigma$ parameter uncertainties are quoted in units of last digit. Tables for isotopically substituted species ${ }^{\mathrm{x}} \mathrm{NiH}$ are given as supplementary material.

\begin{tabular}{|c|c|c|c|c|c|c|}
\hline State & \multicolumn{2}{|c|}{$2 \Delta$} & \multicolumn{2}{|c|}{${ }^{2} \Pi$} & \multicolumn{2}{|c|}{$2 \Sigma^{+}$} \\
\hline Parameter & $\mathrm{NiH}$ & $\mathrm{NiD}$ & $\mathrm{NiH}$ & NiD & $\mathrm{NiH}$ & NiD \\
\hline$T_{\mathrm{e}}$ & 0.0 (fixed) & 0.0 & $2289.568(40)$ & 2267.470 & $1961.242(15)$ & 1937.179 \\
\hline$Y_{10}$ & 1995.651(20) & 1423.6797 & $1892.674(32)$ & 1350.2169 & $1857.622(13)$ & 1325.2111 \\
\hline$Y_{20}$ & $-37.4828(52)$ & -19.07601 & -37.5 (fixed) & -19.085 & -37.5 (fixed) & -19.085 \\
\hline$Y_{30}$ & $0.09154(84)$ & 0.033235 & & & & \\
\hline$Y_{01}$ & $8.0253(13)$ & 4.08429 & $7.48796(40)$ & 3.810824 & $7.6052(16)$ & 3.87049 \\
\hline$Y_{11}$ & $-0.21659(78)$ & -0.078636 & $-0.22442(15)$ & -0.0814788 & $-0.23931(74)$ & -0.086885 \\
\hline $10^{3} Y_{21}$ & $-8.30(11)$ & -2.150 & & & & \\
\hline $10^{4} Y_{02}$ & $-4.7723(100)$ & $-1.2878(26)$ & $-5.229(47)$ & -1.3543 & -4.70 (fixed) & -1.217 \\
\hline $10^{6} Y_{12}$ & $5.14(40)$ & 0.9497 & & & & \\
\hline$A_{\mathrm{e}}$ & -603 (fixed) & -603 & -603 (fixed) & -603 & & \\
\hline$A_{1}$ & $7.033(17)$ & 5.0173 & & & & \\
\hline$\gamma_{\mathrm{e}}$ & $0.950(13)$ & 0.4835 & $1.242(41)$ & 0.63209 & $-0.487(21)$ & -0.24785 \\
\hline$\gamma_{1}$ & $-0.6368(57)$ & -0.23120 & & & & \\
\hline $10^{3} \gamma_{D}$ & $0.149(79)$ & 0.03859 & $0.54(28)$ & 0.140 & $4.50(13)$ & 1.166 \\
\hline$p_{e}$ & $-0.00081(3)$ & -0.000107 & $-0.443(22)$ & -0.2255 & & \\
\hline $10^{3} p_{1}$ & $1.913(28)$ & 0.17989 & & & & \\
\hline $10^{3} p_{D}$ & $-0.00362(14)$ & -0.0002428 & $3.26(11)$ & 0.8444 & & \\
\hline $10^{3} q_{e}$ & $0.0143(14)$ & 0.0009593 & $-23.4(13)$ & -6.061 & & \\
\hline $10^{4} q_{D}$ & & & $-1.499(75)$ & -0.19759 & & \\
\hline$\delta_{00}^{H}$ & 0 (fixed) & & -45 (fixed) & & -49 (fixed) & \\
\hline $10^{5} \delta_{02}^{H}$ & $-4.07(14)$ & & & & & \\
\hline
\end{tabular}


Table 6a Vibrational overlap integrals $f_{\mathrm{v}_{\Delta}, \mathrm{v}_{\Pi}}$ and $f_{\mathrm{v}_{\Pi}, v_{\Sigma}}$ (see table 3) multiplying spin-orbit parameters for ${ }^{58} \mathrm{NiH}$ and ${ }^{58} \mathrm{NiD}$. Most were constrained to 'Initial' values (see text). $1 \sigma$ parameter uncertainties are quoted in units of last digit for those optimized in the fitting procedure. Tables for isotopically substituted species are given as supplementary material.

\begin{tabular}{|c|c|c|c|c|c|c|c|c|}
\hline \multirow{3}{*}{ Parameter } & \multicolumn{4}{|c|}{${ }^{2} \Delta \sim{ }^{2} \Pi$} & \multicolumn{4}{|c|}{${ }^{2} \Pi \sim^{2} \Sigma^{+}$} \\
\hline & \multicolumn{2}{|c|}{$\mathrm{NiH}$} & \multicolumn{2}{|c|}{ NiD } & \multicolumn{2}{|c|}{$\mathrm{NiH}$} & \multicolumn{2}{|c|}{$\mathrm{NiD}$} \\
\hline & Intial & Optimized & Initial & Optimized & Initial & Optimized & Initial & Optimized \\
\hline$f_{0,0}$ & 0.956 & $0.96298(9)$ & 0.940 & $0.95059(12)$ & 0.997 & & 0.996 & \\
\hline$f_{0,1}$ & 0.274 & & 0.318 & & -0.069 & & -0.082 & \\
\hline$f_{0,2}$ & 0.0 & & 0.114 & & 0.0 & & -0.017 & \\
\hline$f_{1,0}$ & -0.293 & $-0.29468(5)$ & -0.253 & $-0.30785(9)$ & 0.067 & & 0.080 & \\
\hline$f_{1,1}$ & 0.869 & $0.89369(8)$ & 0.920 & $0.85476(8)$ & 0.993 & & 0.990 & \\
\hline$f_{1,2}$ & 0.359 & & 0.409 & & -0.095 & & -0.115 & \\
\hline$f_{1,3}$ & 0.0 & & 0.181 & & 0.0 & & -0.031 & \\
\hline$f_{2,0}$ & 0.0 & & 0.044 & & 0.0 & & 0.026 & \\
\hline$f_{2,1}$ & -0.409 & $-0.41153(5)$ & -0.465 & $-0.42880(9)$ & 0.089 & & 0.107 & \\
\hline$f_{2,2}$ & 0.783 & $0.81576(8)$ & 0.710 & $0.75362(26)$ & 0.988 & & 0.983 & \\
\hline$f_{2,3}$ & 0.405 & & 0.451 & & -0.113 & & -0.138 & \\
\hline$f_{2,4}$ & 0.0 & & 0.233 & & 0.0 & & -0.044 & \\
\hline$f_{3,1}$ & 0.0 & & 0.076 & & 0.0 & & 0.043 & \\
\hline$f_{3,2}$ & -0.496 & & -0.552 & $-0.5376(27)$ & 0.101 & & 0.125 & \\
\hline$f_{3,3}$ & 0.699 & & 0.602 & & 0.984 & & 0.977 & \\
\hline$f_{3,4}$ & 0.427 & & 0.466 & & -0.124 & & -0.156 & \\
\hline$f_{3,5}$ & 0.051 & & 0.108 & & 0.0 & & 0.058 & \\
\hline$f_{4,2}$ & -0.565 & & -0.616 & & 0.107 & & 0.136 & \\
\hline$f_{4,3}$ & 0.615 & & 0.498 & & 0.980 & & 0.971 & \\
\hline$f_{4,4}$ & 0.0 & & 0.462 & & 0.0 & & -0.170 & \\
\hline$f_{4,5}$ & 0.0 & & 0.273 & & 0.0 & & -0.058 & \\
\hline$f_{5,3}$ & 0.0 & & 0.140 & & 0.0 & & 0.073 & \\
\hline$f_{5-4}$ & 0.0 & & -0.666 & & 0.0 & & 0.142 & \\
\hline$f_{5,5}$ & 0.0 & & 0.400 & & 0.0 & & 0.966 & \\
\hline
\end{tabular}


Table 6b. Optimized and constrained off-diagonal rotational parameters $B_{\mathrm{v}_{\Delta}, \mathrm{v}_{\Pi}}$ and $B_{\mathrm{v}_{\Pi}, \mathrm{v}_{\Sigma}}\left(\mathrm{in}_{\mathrm{cm}}{ }^{-1}\right)$ for ${ }^{58} \mathrm{NiH}$ and ${ }^{58} \mathrm{NiD}$. Most were constrained to 'Initial' values (see text). $1 \sigma$ parameter uncertainties are quoted in units of last digit for those optimized in the fitting procedure. Tables for isotopically substituted species are given as supplementary material.

\begin{tabular}{|c|c|c|c|c|c|c|c|c|}
\hline \multirow{3}{*}{ Parameter } & \multicolumn{4}{|c|}{${ }^{2} \Delta \sim^{2} \Pi$} & \multicolumn{4}{|c|}{${ }^{2} \Pi \sim^{2} \Sigma^{+}$} \\
\hline & \multicolumn{2}{|r|}{$\mathrm{NiH}$} & \multicolumn{2}{|r|}{$\mathrm{NiD}$} & \multicolumn{2}{|c|}{$\mathrm{NiH}$} & \multicolumn{2}{|c|}{$\mathrm{NiD}$} \\
\hline & Intial & Optimized & Initial & Optimized & Initial & Optimized & Initial & Optimized \\
\hline$B_{0,0}$ & 7.292 & $7.653(31)$ & 3.666 & $3.525(35)$ & 7.392 & $7.3383(27)$ & 3.777 & \\
\hline$B_{0,1}$ & 2.938 & & 1.602 & & 0.408 & & 0.088 & \\
\hline$B_{0,2}$ & 0.0 & & 0.683 & & 0.0 & & -0.030 & \\
\hline$B_{1,0}$ & -1.278 & $-1.4157(34)$ & -1.041 & $-0.976(13)$ & 1.405 & & 0.698 & \\
\hline$B_{1,1}$ & 6.403 & $7.021(18)$ & 3.173 & $3.338(23)$ & 7.102 & $7.0726(26)$ & 3.659 & \\
\hline$B_{1,2}$ & 3.713 & & 2.009 & & 0.559 & & 0.120 & \\
\hline$B_{1,3}$ & 0.0 & & 1.061 & & 0.0 & & -0.053 & \\
\hline$B_{2,0}$ & 0.0 & & 0.049 & & 0.0 & & 0.219 & \\
\hline$B_{2,1}$ & -1.757 & $-1.7922(25)$ & -1.234 & $-1.344(14)$ & 1.871 & & 0.940 & \\
\hline$B_{2,2}$ & 5.558 & $6.1156(84)$ & 2.629 & $2.949(18)$ & 6.812 & & 3.541 & \\
\hline$B_{2,3}$ & 4.032 & & 2.164 & & 0.664 & & 0.144 & \\
\hline$B_{2,4}$ & 0.0 & & 1.336 & & 0.0 & & -0.075 & \\
\hline$B_{3,1}$ & 0.0 & & 0.084 & & 0.0 & & 0.360 & \\
\hline$B_{3,2}$ & -2.088 & & -1.441 & $-1.7309(49)$ & 2.150 & & 1.095 & \\
\hline$B_{3,3}$ & 4.758 & & 2.163 & & 6.525 & & 3.425 & \\
\hline$B_{3,4}$ & 4.094 & & 2.178 & & 0.746 & & 0.163 & \\
\hline$B_{3,5}$ & 0.0 & & 1.526 & & 0.0 & & -0.097 & \\
\hline$B_{4,2}$ & 0.0 & & 0.118 & & 0.0 & & 0.483 & \\
\hline$B_{4,3}$ & -2.334 & & -1.584 & & 2.321 & & 1.200 & \\
\hline$B_{4,4}$ & 4.004 & & 1.731 & & 6.240 & & 3.310 & \\
\hline$B_{4,5}$ & 0.0 & & 2.101 & & 0.0 & & 0.179 & \\
\hline$B_{5,3}$ & 0.0 & & 0.152 & & 0.0 & & 0.591 & \\
\hline$B_{5,4}$ & 0.0 & & -1.681 & & 0.0 & & 1.269 & \\
\hline$B_{5,5}$ & 0.0 & & 1.334 & & 0.0 & & 3.195 & \\
\hline
\end{tabular}


Table 7. Predicted energy terms $\left(\mathrm{cm}^{-1}\right)$ for the lowest rotational levels of NiD ar ${ }^{2} \Pi$ and ${ }^{2} \Sigma^{+}$'supermultiplet' states) $\mathrm{E}<8000 \mathrm{~cm}^{-1}$ ). A more extensive list is given a material. The ${ }^{2} \Lambda$ labels indicate only the dominant contribution to the wavefuncti splittings indicate severe mixing between states.

\begin{tabular}{|c|c|c|c|c|c|c|c|}
\hline \multirow[b]{2}{*}{$J$} & \multicolumn{3}{|c|}{$\mathrm{NiD}$} & \multirow[b]{2}{*}{$\begin{array}{l}\text { State } \\
\text { Label }\end{array}$} & & \multicolumn{2}{|c|}{$\mathrm{NiH}$} \\
\hline & $\begin{array}{r}E_{\text {calc }}, \\
\text { e parity }\end{array}$ & $\begin{array}{r}E_{\text {calc, }} \\
\text { f parity }\end{array}$ & $v$ & & $J$ & $\begin{array}{r}E_{\text {calc, }} \\
\text { e parity }\end{array}$ & $\begin{array}{l}E_{\text {calc, }}, 1 \\
\text { parity }\end{array}$ \\
\hline 0.5 & 2072.72 & 2092.62 & 0 & ${ }^{2} \Sigma^{+}$ & 0.5 & 2052.53 & 2091.15 \\
\hline 0.5 & 3350.78 & 3366.27 & 1 & ${ }^{2} \Sigma^{+}$ & 0.5 & 3477.04 & 3454.22 \\
\hline 0.5 & 3515.49 & 3507.16 & 0 & ${ }^{2} \Pi_{1 / 2}$ & 0.5 & 3851.61 & 3888.02 \\
\hline 0.5 & 4601.43 & 4616.65 & 2 & ${ }^{2} \Sigma^{+}$ & 0.5 & 5275.97 & 5255.37 \\
\hline 0.5 & 4825.18 & 4816.94 & 1 & ${ }^{2} \Pi_{1 / 2}$ & 0.5 & 5577.18 & 5610.88 \\
\hline 0.5 & 5817.60 & 5832.78 & 3 & ${ }^{2} \Sigma^{+}$ & 0.5 & 6996.64 & 6979.43 \\
\hline 0.5 & 6092.93 & 6084.58 & 2 & ${ }^{2} \Pi_{1 / 2}$ & 0.5 & 7231.05 & 7260.77 \\
\hline 0.5 & 6998.26 & 7013.30 & 4 & ${ }^{2} \Sigma^{+}$ & & & \\
\hline 0.5 & 7320.47 & 7312.05 & 3 & ${ }^{2} \Pi_{1 / 2}$ & & & \\
\hline 0.5 & 8145.10 & 8159.63 & 5 & ${ }^{2} \Sigma^{+}$ & & & \\
\hline 1.5 & 973.78 & 973.78 & 0 & ${ }^{2} \Pi_{3 / 2}$ & 1.5 & 2055.23 & 2132.27 \\
\hline 1.5 & 2074.03 & 2113.78 & 0 & ${ }^{2} \Sigma^{+}$ & 1.5 & 2609.71 & 2609.83 \\
\hline 1.5 & 2292.32 & 2292.35 & 1 & ${ }^{2} \Delta_{3 / 2}$ & 1.5 & 2998.95 & 2998.99 \\
\hline 1.5 & 2802.68 & 2802.69 & 0 & ${ }^{2} \Pi_{3 / 2}$ & 1.5 & 3511.01 & 3465.42 \\
\hline 1.5 & 3353.63 & 3384.45 & 1 & ${ }^{2} \Sigma^{+}$ & 1.5 & 3854.88 & 3927.47 \\
\hline 1.5 & 3531.46 & 3514.89 & 0 & ${ }^{2} \Pi_{1 / 2}$ & 1.5 & 4371.23 & 4371.37 \\
\hline 1.5 & 3582.23 & 3582.29 & 2 & ${ }^{2} \Delta_{3 / 2}$ & 1.5 & 4898.28 & 4898.33 \\
\hline 1.5 & 4170.70 & 4170.71 & 1 & ${ }^{2} \Pi_{3 / 2}$ & 1.5 & 5308.03 & 5266.97 \\
\hline 1.5 & 4604.27 & 4634.63 & 2 & ${ }^{2} \Sigma^{+}$ & 1.5 & 5581.36 & 5648.46 \\
\hline 1.5 & 4837.92 & 4839.62 & 3 & ${ }^{2} \Delta_{3 / 2}$ & 1.5 & 6077.53 & 6077.66 \\
\hline 1.5 & 4842.00 & 4823.92 & 1 & ${ }^{2} \Pi_{1 / 2}$ & 1.5 & 6715.96 & 6716.01 \\
\hline 1.5 & 5510.71 & 5510.72 & 2 & ${ }^{2} \Pi_{3 / 2}$ & 1.5 & 7025.93 & 6991.83 \\
\hline 1.5 & 5820.27 & 5850.56 & 3 & ${ }^{2} \Sigma^{+}$ & 1.5 & 7237.02 & 7295.97 \\
\hline 1.5 & 6049.73 & 6049.70 & 4 & ${ }^{2} \Delta_{3 / 2}$ & 1.5 & 7708.24 & 7708.38 \\
\hline 1.5 & 6108.30 & 6091.69 & 2 & ${ }^{2} \Pi_{1 / 2}$ & 1.5 & 8461.20 & 8461.25 \\
\hline 1.5 & 6796.55 & 6796.57 & 3 & ${ }^{2} \Pi_{3 / 2}$ & & & \\
\hline 1.5 & 7000.84 & 7030.83 & 4 & ${ }^{2} \Sigma^{+}$ & & & \\
\hline 1.5 & 7264.20 & 7264.19 & 4 & ${ }^{2} \Pi_{3 / 2}$ & & & \\
\hline 1.5 & 7335.54 & 7318.74 & 3 & ${ }^{2} \Pi_{1 / 2}$ & & & \\
\hline 1.5 & 8052.26 & 8052.31 & 5 & ${ }^{2} \Delta_{3 / 2}$ & & & \\
\hline 2.5 & 0.00 & 0.00 & 0 & ${ }^{2} \Delta_{5 / 2}$ & 2.5 & 0.00 & 0.00 \\
\hline 2.5 & 993.74 & 993.76 & 0 & ${ }^{2} \Delta_{3 / 2}$ & 2.5 & 1011.68 & 1011.83 \\
\hline 2.5 & 1389.92 & 1389.92 & 1 & ${ }^{2} \Delta_{5 / 2}$ & 2.5 & 1925.96 & 1925.96 \\
\hline 2.5 & 2082.87 & 2142.37 & 0 & ${ }^{2} \Sigma^{+}$ & 2.5 & 2072.72 & 2187.79 \\
\hline 2.5 & 2312.03 & 2312.17 & 1 & ${ }^{2} \Delta_{3 / 2}$ & 2.5 & 2648.44 & 2648.95 \\
\hline 2.5 & 2741.98 & 2741.98 & 2 & ${ }^{2} \Delta_{5 / 2}$ & 2.5 & 3036.16 & 3036.32 \\
\hline 2.5 & 2821.93 & 2821.97 & 0 & ${ }^{2} \Pi_{3 / 2}$ & 2.5 & 3559.96 & 3491.71 \\
\hline
\end{tabular}




\begin{tabular}{|c|c|c|c|c|c|c|c|c|c|}
\hline 2.5 & 3363.62 & 3409.45 & 1 & ${ }^{2} \Sigma^{+}$ & 2.5 & 3777.75 & 3777.75 & 2 & ${ }^{2} \Delta_{5 / 2}$ \\
\hline 2.5 & 3555.24 & 3530.62 & 0 & ${ }^{2} \Pi_{1 / 2}$ & 2.5 & 3872.60 & 3980.92 & 1 & ${ }^{2} \Sigma^{+}$ \\
\hline 2.5 & 3601.68 & 3601.92 & 2 & ${ }^{2} \Delta_{3 / 2}$ & 2.5 & 4408.96 & 4409.50 & 1 & ${ }^{2} \Pi_{3 / 2}$ \\
\hline 2.5 & 4056.37 & 4056.37 & 3 & ${ }^{2} \Delta_{5 / 2}$ & 2.5 & 4933.92 & 4934.10 & 2 & ${ }^{2} \Delta_{3 / 2}$ \\
\hline 2.5 & 4189.47 & 4189.50 & 1 & ${ }^{2} \Pi_{3 / 2}$ & 2.5 & 5354.47 & 5293.21 & 1 & ${ }^{2} \Pi_{1 / 2}$ \\
\hline 2.5 & 4614.15 & 4659.46 & 2 & ${ }^{2} \Sigma^{+}$ & 2.5 & 5555.92 & 5555.92 & 3 & ${ }^{2} \Delta_{5 / 2}$ \\
\hline 2.5 & 4857.06 & 4859.39 & 3 & ${ }^{2} \Delta_{3 / 2}$ & 2.5 & 5599.79 & 5699.67 & 2 & ${ }^{2} \Sigma^{+}$ \\
\hline 2.5 & 4865.13 & 4838.44 & 1 & ${ }^{2} \Pi_{1 / 2}$ & 2.5 & 6114.21 & 6114.74 & 2 & ${ }^{2} \Pi_{3 / 2}$ \\
\hline 2.5 & 5333.31 & 5333.31 & 4 & ${ }^{2} \Delta_{5 / 2}$ & 2.5 & 6749.94 & 6750.11 & 3 & ${ }^{2} \Delta_{3 / 2}$ \\
\hline 2.5 & 5528.97 & 5529.01 & 2 & ${ }^{2} \Pi_{3 / 2}$ & 2.5 & 7068.68 & 7018.38 & 2 & ${ }^{2} \Pi_{1 / 2}$ \\
\hline 2.5 & 5829.83 & 5875.07 & 3 & ${ }^{2} \Sigma^{+}$ & 2.5 & 7257.17 & 7344.46 & 3 & ${ }^{2} \Sigma^{+}$ \\
\hline 2.5 & 6068.34 & 6068.20 & 4 & ${ }^{2} \Delta_{3 / 2}$ & 2.5 & 7261.12 & 7260.97 & 4 & ${ }^{2} \Delta_{5 / 2}$ \\
\hline 2.5 & 6131.09 & 6106.45 & 2 & ${ }^{2} \Pi_{1 / 2}$ & 2.5 & 7743.89 & 7744.44 & 3 & ${ }^{2} \Pi_{3 / 2}$ \\
\hline 2.5 & 6572.99 & 6572.99 & 5 & ${ }^{2} \Delta_{5 / 2}$ & 2.5 & 8493.19 & 8493.38 & 4 & ${ }^{2} \Delta_{3 / 2}$ \\
\hline 2.5 & 6814.34 & 6814.40 & 3 & ${ }^{2} \Pi_{3 / 2}$ & 2.5 & 8894.05 & 8894.05 & 5 & ${ }^{2} \Delta_{5 / 2}$ \\
\hline 2.5 & 7010.19 & 7055.00 & 4 & ${ }^{2} \Sigma^{+}$ & & & & & \\
\hline 2.5 & 7282.43 & 7282.42 & 4 & ${ }^{2} \Pi_{3 / 2}$ & & & & & \\
\hline 2.5 & 7357.82 & 7332.77 & 3 & ${ }^{2} \Pi_{1 / 2}$ & & & & & \\
\hline 2.5 & 8069.38 & 8069.59 & 5 & ${ }^{2} \Delta_{3 / 2}$ & & & & & \\
\hline 3.5 & 27.93 & 27.93 & 0 & ${ }^{2} \Delta_{5 / 2}$ & 3.5 & 53.83 & 53.83 & 0 & ${ }^{2} \Delta_{5 / 2}$ \\
\hline 3.5 & 1021.68 & 1021.73 & 0 & ${ }^{2} \Delta_{3 / 2}$ & 3.5 & 1065.95 & 1066.33 & 0 & ${ }^{2} \Delta_{3 / 2}$ \\
\hline 3.5 & 1417.22 & 1417.22 & 1 & ${ }^{2} \Delta_{5 / 2}$ & 3.5 & 1978.01 & 1978.01 & 1 & ${ }^{2} \Delta_{5 / 2}$ \\
\hline 3.5 & 2099.26 & 2178.35 & 0 & ${ }^{2} \Sigma^{+}$ & 3.5 & 2105.07 & 2257.57 & 0 & ${ }^{2} \Sigma^{+}$ \\
\hline 3.5 & 2339.58 & 2339.94 & 1 & ${ }^{2} \Delta_{3 / 2}$ & 3.5 & 2702.52 & 2703.79 & 0 & ${ }^{2} \Pi_{3 / 2}$ \\
\hline 3.5 & 2768.65 & 2768.65 & 2 & ${ }^{2} \Delta_{5 / 2}$ & 3.5 & 3088.17 & 3088.57 & 1 & ${ }^{2} \Delta_{3 / 2}$ \\
\hline 3.5 & 2848.87 & 2848.96 & 0 & ${ }^{2} \Pi_{3 / 2}$ & 3.5 & 3623.82 & 3533.07 & 0 & ${ }^{2} \Pi_{1 / 2}$ \\
\hline 3.5 & 3380.82 & 3441.18 & 1 & ${ }^{2} \Sigma^{+}$ & 3.5 & 3828.01 & 3828.02 & 2 & ${ }^{2} \Delta_{5 / 2}$ \\
\hline 3.5 & 3586.79 & 3554.39 & 0 & ${ }^{2} \Pi_{1 / 2}$ & 3.5 & 3904.90 & 4048.24 & 1 & ${ }^{2} \Sigma^{+}$ \\
\hline 3.5 & 3628.85 & 3629.44 & 2 & ${ }^{2} \Delta_{3 / 2}$ & 3.5 & 4461.64 & 4462.96 & 1 & ${ }^{2} \Pi_{3 / 2}$ \\
\hline 3.5 & 4082.42 & 4082.42 & 3 & ${ }^{2} \Delta_{5 / 2}$ & 3.5 & 4983.72 & 4984.15 & 2 & ${ }^{2} \Delta_{3 / 2}$ \\
\hline 3.5 & 4215.71 & 4215.80 & 1 & ${ }^{2} \Pi_{3 / 2}$ & 3.5 & 5415.12 & 5334.11 & 1 & ${ }^{2} \Pi_{1 / 2}$ \\
\hline 3.5 & 4631.11 & 4691.09 & 2 & ${ }^{2} \Sigma^{+}$ & 3.5 & 5604.38 & 5604.43 & 3 & ${ }^{2} \Delta_{5 / 2}$ \\
\hline 3.5 & 4883.71 & 4887.02 & 3 & ${ }^{2} \Delta_{3 / 2}$ & 3.5 & 5632.70 & 5764.37 & 2 & ${ }^{2} \Sigma^{+}$ \\
\hline 3.5 & 4895.93 & 4860.52 & 1 & ${ }^{2} \Pi_{1 / 2}$ & 3.5 & 6165.41 & 6166.74 & 2 & ${ }^{2} \Pi_{3 / 2}$ \\
\hline 3.5 & 5358.73 & 5358.73 & 4 & ${ }^{2} \Delta_{5 / 2}$ & 3.5 & 6797.39 & 6797.83 & 3 & ${ }^{2} \Delta_{3 / 2}$ \\
\hline 3.5 & 5554.51 & 5554.60 & 2 & ${ }^{2} \Pi_{3 / 2}$ & 3.5 & 7124.48 & 7059.15 & 2 & ${ }^{2} \Pi_{1 / 2}$ \\
\hline 3.5 & 5846.32 & 5906.28 & 3 & ${ }^{2} \Sigma^{+}$ & 3.5 & 7292.21 & 7406.07 & 3 & ${ }^{2} \Sigma^{+}$ \\
\hline 3.5 & 6094.37 & 6093.95 & 4 & ${ }^{2} \Delta_{3 / 2}$ & 3.5 & 7307.83 & 7307.66 & 4 & ${ }^{2} \Delta_{5 / 2}$ \\
\hline 3.5 & 6161.27 & 6128.95 & 2 & ${ }^{2} \Pi_{1 / 2}$ & 3.5 & 7793.65 & 7795.01 & 3 & ${ }^{2} \Pi_{3 / 2}$ \\
\hline 3.5 & 6597.78 & 6597.78 & 5 & ${ }^{2} \Delta_{5 / 2}$ & 3.5 & 8746.09 & 8707.99 & 3 & ${ }^{2} \Pi_{1 / 2}$ \\
\hline 3.5 & 6839.19 & 6839.37 & 3 & ${ }^{2} \Pi_{3 / 2}$ & 3.5 & 8893.62 & 8977.77 & 4 & ${ }^{2} \Sigma^{+}$ \\
\hline 3.5 & 7026.36 & 7085.78 & 4 & ${ }^{2} \Sigma^{+}$ & 3.5 & 8939.69 & 8939.69 & 5 & ${ }^{2} \Delta_{5 / 2}$ \\
\hline 3.5 & 7307.93 & 7307.86 & 4 & ${ }^{2} \Pi_{3 / 2}$ & & & & & \\
\hline 3.5 & 7387.28 & 7354.18 & 3 & ${ }^{2} \Pi_{1 / 2}$ & & & & & \\
\hline 3.5 & 8093.23 & 8093.78 & 5 & ${ }^{2} \Delta_{3 / 2}$ & & & & & \\
\hline 4.5 & 63.82 & 63.82 & 0 & ${ }^{2} \Delta_{5 / 2}$ & 4.5 & 122.96 & 122.96 & 0 & ${ }^{2} \Delta_{5 / 2}$ \\
\hline 4.5 & 1057.57 & 1057.67 & 0 & ${ }^{2} \Delta_{3 / 2}$ & 4.5 & 1135.59 & 1136.36 & 0 & ${ }^{2} \Delta_{3 / 2}$ \\
\hline 4.5 & 1452.30 & 1452.30 & 1 & ${ }^{2} \Delta_{5 / 2}$ & 4.5 & 2044.85 & 2044.86 & 1 & ${ }^{2} \Delta_{5 / 2}$ \\
\hline 4.5 & 2123.21 & 2221.69 & 0 & ${ }^{2} \Sigma^{+}$ & 4.5 & 2152.31 & 2341.47 & 0 & ${ }^{2} \Sigma^{+}$ \\
\hline
\end{tabular}




\begin{tabular}{llllllllll}
\hline 4.5 & 2374.96 & 2375.68 & 1 & ${ }^{2} \Delta_{3 / 2}$ & 4.5 & 2771.86 & 2774.39 & 0 & ${ }^{2} \Pi_{3 / 2}$ \\
4.5 & 2802.91 & 2802.91 & 2 & ${ }^{2} \Delta_{5 / 2}$ & 4.5 & 3154.89 & 3155.70 & 1 & ${ }^{2} \Delta_{3 / 2}$ \\
4.5 & 2883.47 & 2883.66 & 0 & ${ }^{2} \Pi_{3 / 2}$ & 4.5 & 3702.51 & 3589.50 & 0 & ${ }^{2} \Pi_{1 / 2}$ \\
4.5 & 3405.26 & 3479.55 & 1 & ${ }^{2} \Sigma^{+}$ & 4.5 & 3892.55 & 3892.59 & 2 & ${ }^{2} \Delta_{5 / 2}$ \\
4.5 & 3626.07 & 3586.22 & 0 & ${ }^{2} \Pi_{1 / 2}$ & 4.5 & 3951.87 & 4129.28 & 1 & ${ }^{2} \Sigma^{+}$ \\
4.5 & 3663.72 & 3664.89 & 2 & ${ }^{2} \Delta_{3 / 2}$ & 4.5 & 4529.16 & 4531.79 & 1 & ${ }^{2} \Pi_{3 / 2}$ \\
4.5 & 4115.88 & 4115.88 & 3 & ${ }^{2} \Delta_{5 / 2}$ & 4.5 & 5047.59 & 5048.45 & 2 & ${ }^{2} \Delta_{3 / 2}$ \\
4.5 & 4249.43 & 4249.59 & 1 & ${ }^{2} \Pi_{3 / 2}$ & 4.5 & 5489.75 & 5389.68 & 1 & ${ }^{2} \Pi_{1 / 2}$ \\
4.5 & 4655.16 & 4729.47 & 2 & ${ }^{2} \Sigma^{+}$ & 4.5 & 5666.44 & 5666.73 & 3 & ${ }^{2} \Delta_{5 / 2}$ \\
4.5 & 4917.91 & 4922.52 & 3 & ${ }^{2} \Delta_{3 / 2}$ & 4.5 & 5680.49 & 5842.41 & 2 & ${ }^{2} \Sigma^{+}$ \\
4.5 & 4934.29 & 4890.14 & 1 & ${ }^{2} \Pi_{1 / 2}$ & 4.5 & 6231.03 & 6233.66 & 2 & ${ }^{2} \Pi_{3 / 2}$ \\
4.5 & 5391.39 & 5391.39 & 4 & ${ }^{2} \Delta_{5 / 2}$ & 4.5 & 6858.21 & 6859.11 & 3 & ${ }^{2} \Delta_{3 / 2}$ \\
4.5 & 5587.31 & 5587.49 & 2 & ${ }^{2} \Pi_{3 / 2}$ & 4.5 & 7192.77 & 7114.16 & 2 & ${ }^{2} \Pi_{1 / 2}$ \\
4.5 & 5869.77 & 5944.15 & 3 & ${ }^{2} \Sigma^{+}$ & 4.5 & 7342.51 & 7480.63 & 3 & ${ }^{2} \Sigma^{+}$ \\
4.5 & 6127.79 & 6126.77 & 4 & ${ }^{2} \Delta_{3 / 2}$ & 4.5 & 7367.90 & 7367.60 & 4 & ${ }^{2} \Delta_{5 / 2}$ \\
4.5 & 6198.82 & 6159.39 & 2 & ${ }^{2} \Pi_{1 / 2}$ & 4.5 & 7857.40 & 7860.10 & 3 & ${ }^{2} \Pi_{3 / 2}$ \\
4.5 & 6629.65 & 6629.65 & 5 & ${ }^{2} \Delta_{5 / 2}$ & 4.5 & 8595.00 & 8596.01 & 4 & ${ }^{2} \Delta_{3 / 2}$ \\
4.5 & 6871.10 & 6871.45 & 3 & ${ }^{2} \Pi_{3 / 2}$ & 4.5 & 8806.22 & 9049.31 & 4 & ${ }^{2} \Sigma^{+}$ \\
4.5 & 7049.37 & 7123.12 & 4 & ${ }^{2} \Sigma^{+}$ & 4.5 & 8949.86 & 8763.48 & 3 & ${ }^{2} \Pi_{1 / 2}$ \\
4.5 & 7340.66 & 7340.43 & 4 & ${ }^{2} \Pi_{3 / 2}$ & 4.5 & 8998.31 & 8998.31 & 5 & ${ }^{2} \Delta_{5 / 2}$ \\
4.5 & 7423.91 & 7383.06 & 3 & ${ }^{2} \Pi_{1 / 2}$ & & & & & \\
4.5 & 8123.73 & 8124.90 & 5 & ${ }^{2} \Delta_{3 / 2}$ & & & & & \\
\hline
\end{tabular}




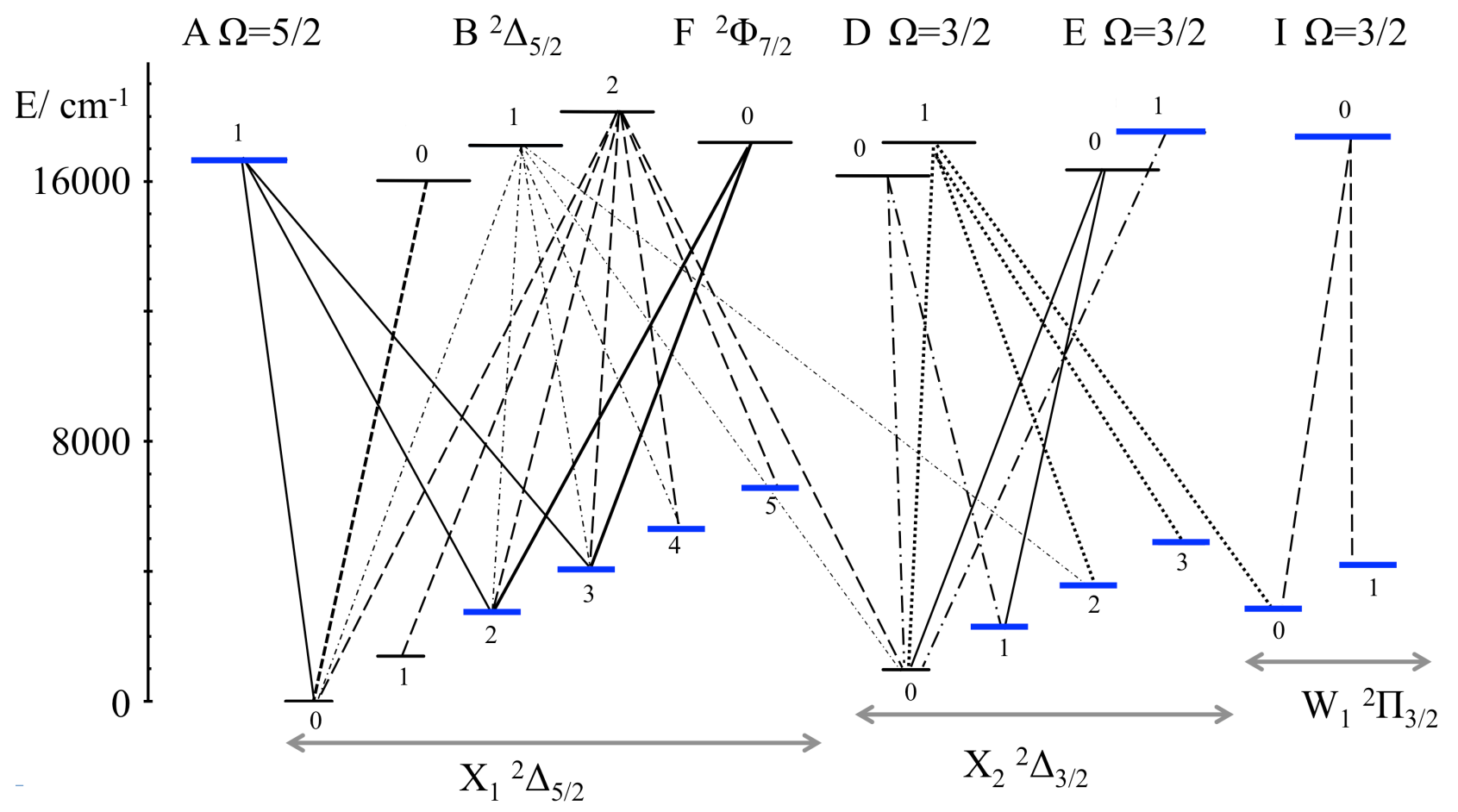

FIG. 1. (colour online) Emission bands observed in laser-induced fluoresence spectra of NiD. Thicker (blue) lines indicate hitherto unobserved vibrational levels in these electronic states. 

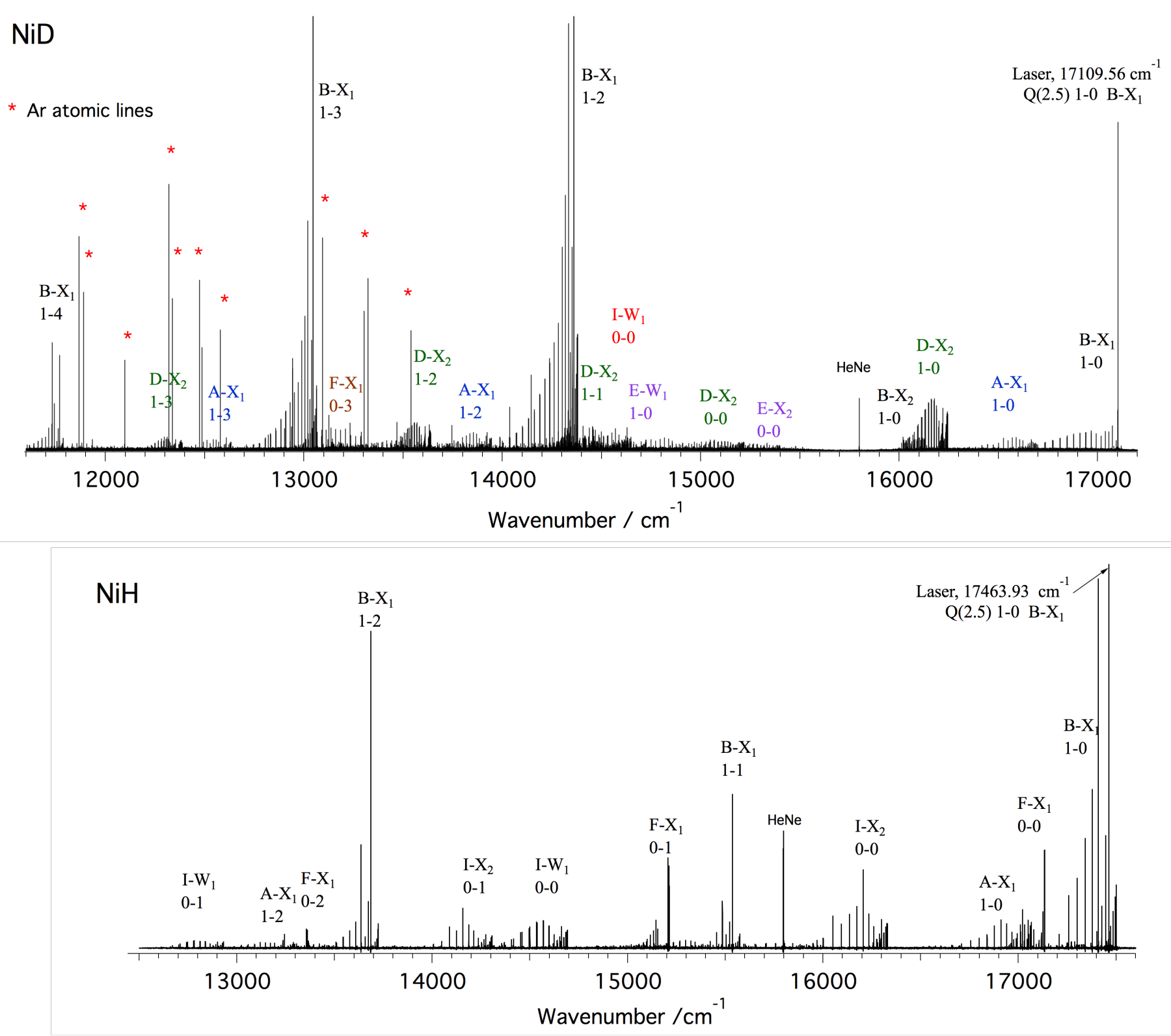

Figure 2. (colour online) Resolved fluorescence spectra of $\mathrm{NiD}$ and $\mathrm{NiH}$ (resolution $0.04 \mathrm{~cm}^{-1}$ ) showing collisionally-induced transitions from several electronic systems. In both cases, the laser excited $\mathrm{Q}_{\mathrm{ef}, \mathrm{fe}}(2.5) \mathrm{B}-\mathrm{X}_{1}(1-0)$. Some lines (notably around $14800 \mathrm{~cm}^{-1}$ ) remain unassigned in the NiD spectrum. The Ar lines (*) are also present in the NiH spectrum, but are much weaker than the molecular emission. 


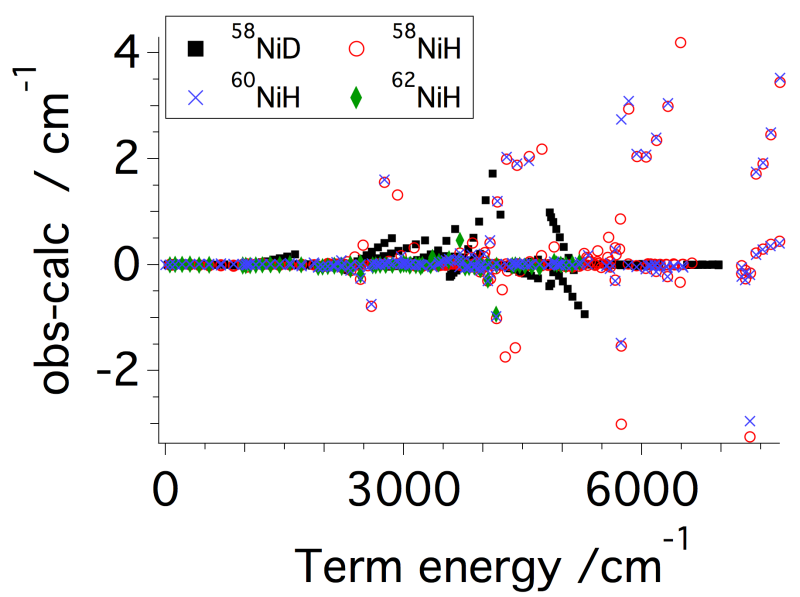

(a)

(b)

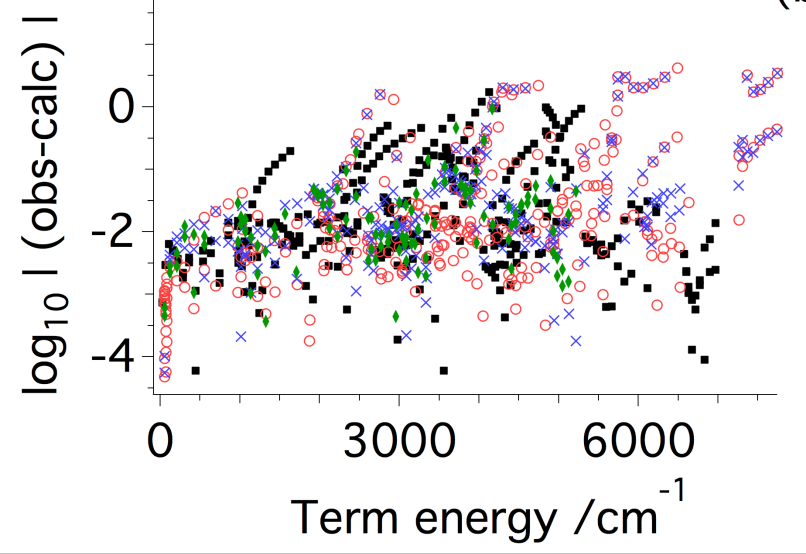

Figure 3. (colour online) Distribution of obs-calc residuals generated from the multi-isotope fit. The logarithmic scale in panel (b) illustrates more clearly the distribution of residuals $<0.05 \mathrm{~cm}^{-1}$, indistinguishable in panel (a). 
Figure 4. (colour online) Comparison between predicted term values for ${ }^{58} \mathrm{NiD}$ at $J=2.5$ from our multi-isotope fit (thick lines), and from ab initio calculations of C. Marian [12], (thinner (blue) lines). Labels indicate the dominant wavefunction contribution ${ }^{2} \Lambda_{\Omega}$ and vibrational levels.

Connectors (red) link predictions for the $\Omega=1 / 2$ states. Asterisks indicate experimentally observed levels.

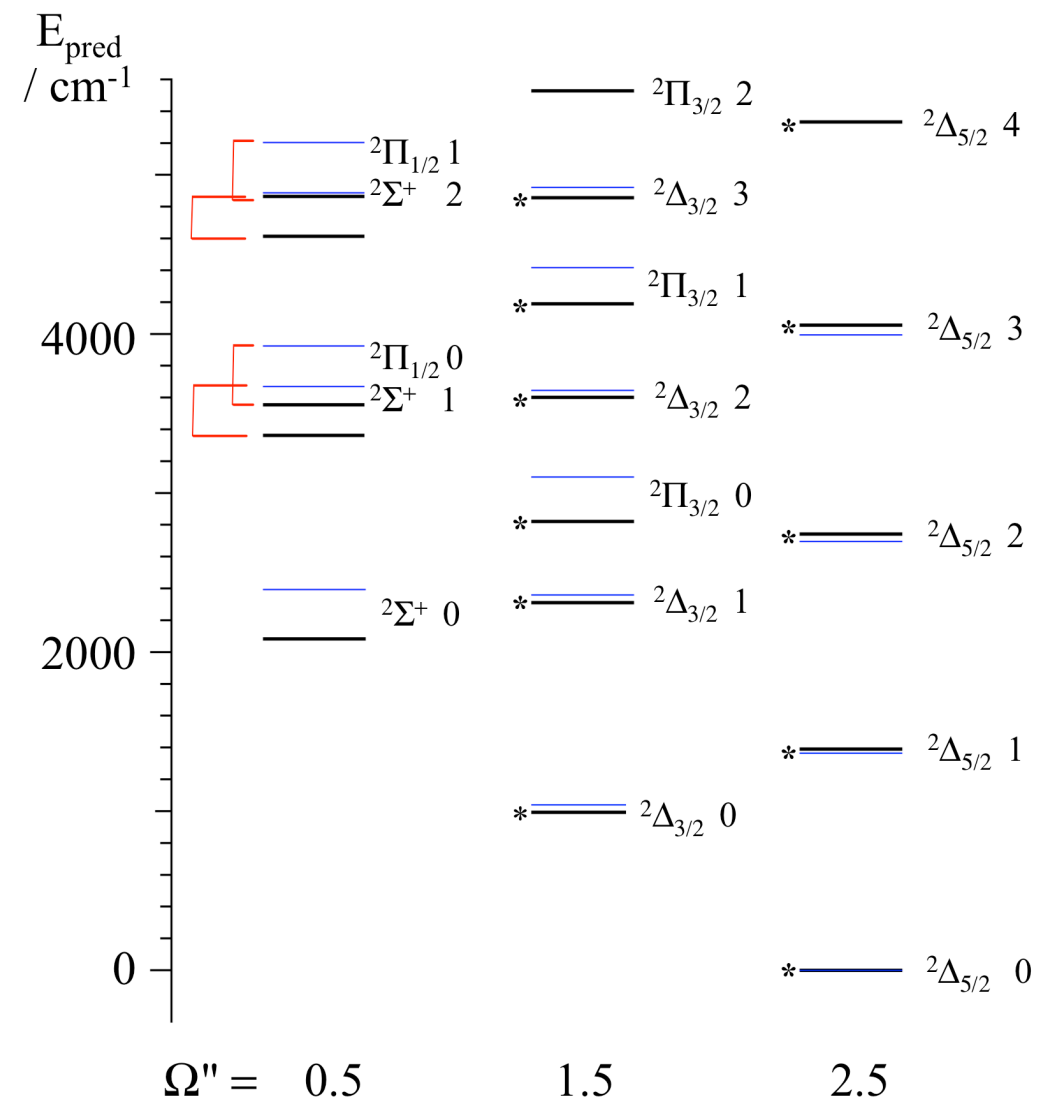




\section{References}

[1] S.R. Langhoff and C.W. Bauschlicher, Ann. Rev. Phys. Chem. 39, 181 (1988).

[2] M.R.A. Blomberg, P.E.M. Seigbahn, and B.O. Roos, Mol. Phys. 47, 127 (1982).

[3] B.I. Dunlap and H.L. Yu, Chem. Phys. Lett. 73 (3), 525 (1980).

[4] M.P. Guse, R.J. Blint, and A.B. Kunz, International Journal of Quantum Chemistry 11 (5), 725 (1977).

[5] N.J. Mayhall and K. Raghavachari, J. Chem. Theo. \& Comp. 6 (9), 2714 (2010).

[6] P.S. Bagus and C. Bjorkman, Phys. Rev. A 23 (2), 461 (1981).

[7] S.P. Walch and C.W. Bauschlicher, Chem. Phys. Lett. 86 (1), 66 (1982).

[8] S.P. Walch and C.W. Bauschlicher, J. Chem. Phys. 78 (7), 4597 (1983).

[9] F. Ruette, G. Blyholder, and J. Head, J. Chem. Phys. 80 (5), 2042 (1984).

[10] R. Pouamerigo, M. Merchan, I. Nebotgil, P.A. Malmqvist, and B.O. Roos, J. Chem. Phys. 101 (6), 4893 (1994).

[11] C.M. Marian, J. Chem. Phys. 93 (2), 1176 (1990).

[12] C.M. Marian, Ber. Bunsen Phys. Chem. 99 (3), 254 (1995).

[13] W.L. Zou and W.J. Liu, J. Comp. Chem. 28 (14), 2286 (2007).

[14] J.H. Chen and T.C. Steimle, Chem. Phys. Lett. 457 (1-3), 23 (2008).

[15] S.P. Walch, C.W. Bauschlicher, and S.R. Langhoff, J. Chem. Phys. 83 (10), 5351 (1985).

[16] D.P. Chong, S.R. Langhoff, C.W. Bauschlicher, and S.P. Walch, J. Chem. Phys. 85 (5), 2850 (1986).

[17] C.W. Bauschlicher, S.R. Langhoff, and A. Komornicki, Theoretica Chimica Acta 77 (4), 263 (1990).

[18] C.V. Diaconu, A.E. Cho, J.D. Doll, and D.L. Freeman, J. Chem. Phys. 121 (20), 10026 (2004). 
[19] S. Goel and A.E. Masunov, J. Chem. Phys. 129 (21), 214302 (2008).

[20] A.G. Gaydon and R.W.B. Pearse, Proc. Roy. Soc. London A 148, 312 (1935).

[21] A. Heimer, Z. Phys. 105 (1), 56 (1937).

[22] N. Aslund, H. Neuhaus, Lagerqvist. A, and E. Andersen, Arkiv for Fysik 28 (3), 271 (1965).

[23] T.C. Devore, High Temperature Science 12 (4), 259 (1980).

[24] R. Scullman, S. Löfgren, and S. Adakkai Kadavathu, Phys. Scr. 25 (2), 295 (1982).

[25] S. Adakkai Kadavathu, S. Lofgren, and R. Scullman, Phys. Scr. 35 (3), 277 (1987).

[26] S. Adakkai Kadavathu, R. Scullman, R.W. Field, J.A. Gray, and M. Li, J. Mol. Spectrosc. 147, 448 (1991).

[27] S. Adakkai Kadavathu, R. Scullman, J.A. Gray, M. Li, and R.W. Field, J. Mol. Spectrosc. 140, 126 (1990).

[28] T. Nelis, S.P. Beaton, K.M. Evenson, and J.M. Brown, J. Mol. Spectrosc. 148, 462 (1991).

[29] S.P. Beaton, K.M. Evenson, T. Nelis, and J.M. Brown, J. Chem. Phys. 89 (7), 4446 (1988).

[30] K. Lipus, U. Simon, E. Bachem, T. Nelis, and W. Urban, Mol. Phys. 67 (6), 1431 (1989).

[31] K. Lipus, E. Bachem, and W. Urban, Mol. Phys. 75 (4), 945 (1992).

[32] K. Lipus, W. Urban, K.M. Evenson, and J.M. Brown, Mol. Phys. 79 (3), 571 (1993).

[33] J.A. Gray and R.W. Field, J. Chem. Phys. 84 (2), 1041 (1986).

[34] C.W. Bauschlicher and B.O. Roos, J. Chem. Phys. 91, 4785 (1989).

[35] M.C. McCarthy, H. Kanamori, T.C. Steimle, M.G. Li, and R.W. Field, J. Chem. Phys. 107 (11), 4179 (1997).

[36] H. Harker, C. Richard, G. Tourasse, P. Crozet, and A.J. Ross, J. Mol. Spectrosc. 292, 28 (2013). 
[37] J.A. Gray, M. Li, T. Nelis, and R.W. Field, J. Chem. Phys. 95, 7164 (1991).

[38] A.J. Ross, P. Crozet, C. Richard, H. Harker, S.H. Ashworth, and D.W. Tokaryk, Mol. Phys. 110 (17), 2019 (2012).

[39] R. Vallon, C. Richard, P. Crozet, G. Wannous, and A. Ross, Astrophys. J. 696 (1), 172 (2009).

[40] R. Vallon, S.H. Ashworth, P. Crozet, R.W. Field, D. Forthomme, H. Harker, C. Richard, and A.J. Ross, J. Phys. Chem. A 113 (47), 13159 (2009).

[41] J.M. Brown, E.A. Colbourn, J.K.G. Watson, and F.D. Wayne, J. Mol. Spectrosc. 74 (2), 294 (1979).

[42] J.M. Brown, A.S.C. Cheung, and A.J. Merer, J. Mol. Spectrosc. 124 (2), 464 (1987).

[43] J.M. Brown and A.J. Merer, J. Mol. Spectrosc. 74 (3), 488 (1979).

[44] H. Lefebvre-Brion and R.W. Field, The spectra and dynamics of diatomic molecules (Elsevier, Amsterdam, 2004), p. 766.

[45] R.J. Le Roy, J. Mol. Spectrosc. 194 (2), 189 (1999).

[46] J.M. Brown and J.K.G. Watson, J. Mol. Spectrosc. 65 (1), 65 (1977).

[47] R.J. Le Roy, J. Quant. Spectrosc. Rad. Trans. 186, 167 (2017).

[48] R.J. Le Roy, J. Quant. Spectrosc. Rad. Trans. 186, 158 (2017).

[49] R.J. Le Roy, J. Quant. Spectrosc. Rad. Transf. 186, 197 (2017).

[50] D.L. Lambert and E.A. Mallia, Mon. Not. Roy. Astron. Soc. 151 (4), 437 (1971).

[51] L.C. O'Brien and J.J. O'Brien, Astrophys. J. 621 (1), 554 (2005).

[52] S. Shaji, J. Nunn, J.J. O'Brien, and L.C. O'Brien, Astrophys. J. 672, 722 (2008).

[53] S. Shaji, A. Song, M. Li, J.J. O'Brien, and L.C. O'Brien, Canad. J. Phys. 87 (5), 583 (2009).

[54] L. Wallace, K. Hinkle, and W. Livingston, NSO technical report ; 00-01 An atlas of sunspot umbral spectra in the visible, from 15,000 to 25,500 cm(-1) (3920 to 6664 [Angstrom]) (2000). 
[55] S.N. Yurchenko, L. Lodi, J. Tennyson, and A.V. Stolyarov, Computer Physics Communications 202, $262(2016)$.

[56] J. Tennyson and S.N. Yurchenko, Mon. Not. Roy. Astron. Soc. 425 (1), 21 (2012).

[57] O. Dulieu and P.S. Julienne, J. Chem. Phys. 103 (1), 60 (1995).

[58] A.N. Drozdova, A.V. Stolyarov, M. Tamanis, R. Ferber, P. Crozet, and A.J. Ross, Phys. Rev. A 88, 022504 (2013).

[59] K. Alps, A. Kruzins, M. Tamanis, R. Ferber, E.A. Pazyuk, and A.V. Stolyarov, J. Chem. Phys. 144 (14), 144310 (2016). 